\title{
The vorticity budget of developing typhoon Nuri (2008)
}

\author{
D. J. Raymond and C. López Carrillo \\ New Mexico Tech, Socorro, New Mexico, USA \\ Received: 20 April 2010 - Published in Atmos. Chem. Phys. Discuss.: 2 July 2010 \\ Revised: 23 November 2010 - Accepted: 10 December 2010 - Published: 10 January 2011
}

\begin{abstract}
The formation of west Pacific tropical cyclone Nuri (2008) was observed over four days from easterly wave to typhoon stage by aircraft using scanning Doppler radar and dropsonde data. This disturbance developed rapidly in a significantly sheared environment. In spite of the shear, overlapping closed circulations existed in the frame of reference of the storm in the planetary boundary layer and at $5 \mathrm{~km}$ elevation, providing a deep region protected from environmental influences. The rapid spinup of Nuri can be attributed to the strong increase with height at low levels of the vertical mass flux during and after the tropical depression stage, and the correspondingly strong vorticity convergence in the planetary boundary layer. As Nuri developed, convective regions of boundary layer vortex stretching became fewer but more intense, culminating in a single nascent eyewall at the tropical storm stage. A non-developing tropical wave case was also analyzed. This system started with much weaker circulations in the boundary layer and aloft, leaving it unprotected against environmental intrusion. This may explain its failure to develop.
\end{abstract}

\section{Introduction}

A detailed accounting of the vorticity budget of a developing tropical cyclone is necessary to understand how the storm spins up. A variety of different mechanisms have been advanced to explain this process.

Developing tropical systems often first exhibit significant cyclonic vorticity at middle levels in the troposphere (Ritchie and Holland, 1997; Simpson et al. (1997); Bister and Emanuel, 1997; Raymond et al., 1998). However, the development of strong near-surface vorticity and the associ-

Correspondence to: D. J. Raymond (raymond@kestrel.nmt.edu) ated warm core is a key element of tropical cyclone spinup. An important problem is then to understand how the surface vortex develops.

Ritchie and Holland (1997) and Simpson et al. (1997) assert that mid-level vortex merger leads to downward vortex development, but do not explain how this might occur. Potential vorticity anomalies with larger horizontal scale exhibit greater vertical penetration. Quasi-geostrophic calculations of this effect for the vortices described in their paper suggest that the increased penetration is minimal, but as Davis (1992) has shown, stronger vortices in the nonlinear balance regime exhibit greater vertical penetration than quasigeostrophic calculations would suggest.

Bister and Emanuel (1997) argue that the development of a cool, moist environment resulting from stratiform rain serves as the incubation region for the formation of a low-level, warm-core vortex. Cloud resolving model calculations by Raymond and Sessions (2007) support this assertion. Environments cooler at low levels and warmer at upper levels by of order $1 \mathrm{~K}$ lower the elevation of maximum vertical mass flux from $\approx 10 \mathrm{~km}$ to $\approx 5 \mathrm{~km}$ in their calculations, and by virtue of mass continuity, intensify the low-level inflow into the convection. In the presence of ambient vorticity, low-level convergence would contribute to the spinup of the system. This effect may explain why tropical-wave-scale mid-level vorticity fosters tropical storm formation. The balanced response to a mid-level vortex is a cold core below the vortex and a warm core above, as assumed by Raymond and Sessions (2007). Mapes and Houze (1995) may have detected this phenomenon in the outer rainbands of a south Pacific tropical cyclone, a region far removed from the cyclone's warm core.

Dunkerton et al. (2009) emphasize the importance of a continuing protected region or "pouch" consisting of a quasiclosed circulation in the low to middle troposphere. This pouch occurs where the critical latitude for a tropical wave (latitude at which the wave propagation speed equals the

Published by Copernicus Publications on behalf of the European Geosciences Union. 
wind speed) intersects the wave trough axis. Near this point the system-relative circulation is approximately closed, which means that dry air from the surrounding environment cannot easily enter and vorticity cannot easily escape. Dunkerton et al. (2009) also emphasized that strong deformation flow impedes the organization of vorticity into a coherent, axially symmetric structure.

Assuming that convergence of low-level vorticity is the most important mechanism for spinning up a tropical cyclone, how much of this convergence is the result of widespread, ordinary convection and how much of it occurs in strong but isolated convective systems? The overall circulation tendency around the cyclone doesn't depend on how vorticity convergence is distributed within the circulation loop, but the internal structure of the system does.

Hendricks et al. (2004) and Montgomery et al. (2006) present evidence that strong, isolated convection prevails, and denote these convective systems vortical hot towers (VHTs). The updrafts of VHTs are rotating rapidly and Hendricks et al. (2004) suggest that this rotation may be rapid enough to suppress entrainment and thus alter the vertical mass flux profile of VHTs compared to more mundane forms of deep tropical convection. Furthermore, the core of the developing tropical cyclone appears to form from the merger of the residual vortices left by VHTs.

Measurements from a recent field program are used here to clarify the vorticity budget during the formation and intensification of typhoon Nuri (2008) and in a non-developing tropical wave. The THORPEX Pacific Asian Regional Campaign (TPARC) and the associated Tropical Cyclone Structure experiment (TCS08) (Elsberry and Harr, 2008) studied various aspects of western Pacific typhoons in August and September of 2008. Numerous observational tools were focused on the formation and development of typhoons and on their extratropical transitions during this period.

Typhoon Nuri (2008) intensified from a tropical wave to a typhoon in three days. Montgomery et al. (2010) present the synoptic conditions in which the precursor tropical wave developed into a tropical cyclone. They show that the core of the cyclone formed at the critical latitude, i.e., the latitude at which the low-level wind equaled the wave propagation speed. This supports the hypothesis of Dunkerton et al. (2009) that the region of the wave at the critical latitude is favored for development due to the weak wave-relative winds and the lack of import of dry environmental air at this latitude. A measure of shear versus curvature vorticity showed that the latter dominated in the favored region during the period of development as well, thus avoiding severe deformation of the vorticity anomaly.

We were able to observe tropical cyclone Nuri on four successive days, later denoted "Nuri 1" through "Nuri 4", with varying combinations of dropsondes and airborne Doppler radar. On these four days the system was successively a tropical wave (TW), a tropical depression (TD), a tropical storm (TS), and a typhoon (TY). The data are sufficient to analyze all terms in the vorticity equation through the full depth of the system on the first three days and in the lowest $3 \mathrm{~km}$ on the fourth day. The absence of Doppler radar data limits the analysis at higher levels on day four. We captured an unusually complete picture of Nuri's intensification from these observations, shedding significant light on the vorticity dynamics of tropical storm formation. We also observed in a single mission a non-developing tropical wave, designated TCS030. The contrast between this system and the earliest observed stage of Nuri is enlightening.

Section 2 covers the theoretical underpinnings of our observational analysis. Section 3 discusses the data sources and analysis methods used in this case study. The vorticity budgets of tropical cyclone Nuri at its various stages and of tropical wave TCS030 are described in Sect. 4. The implications of these results are discussed in Sect. 5 and conclusions are presented in Sect. 6.

\section{Theoretical considerations}

Our analysis of Doppler radar and dropsonde observations is enhanced by an understanding of certain aspects of the vorticity and divergence equations discussed below.

\subsection{Governing equations}

Using the identity $\boldsymbol{v} \cdot \nabla \boldsymbol{v}=\nabla\left(v^{2} / 2\right)-\boldsymbol{v} \times \zeta_{r}$ where $\boldsymbol{v}$ is the wind field, $v=|\boldsymbol{v}|$, and $\zeta_{r}=\boldsymbol{\nabla} \times \boldsymbol{v}$ is the relative vorticity, the horizontal momentum equation may be written

$$
\frac{\partial \boldsymbol{v}_{h}}{\partial t}+\nabla_{h}\left(v^{2} / 2\right)+\hat{\boldsymbol{k}} \times \boldsymbol{Z}+\theta \boldsymbol{\nabla}_{h} \Pi=0
$$

where the subscripted $h$ indicates the horizontal part of a vector, a subscripted $z$ indicates the vertical component, and where

$\boldsymbol{Z}=\boldsymbol{Z}_{1}+\boldsymbol{Z}_{2}+\boldsymbol{Z}_{f}=\boldsymbol{v}_{h} \zeta_{z}-\zeta_{h} v_{z}+\hat{\boldsymbol{k}} \times \boldsymbol{F}$.

The quantity $\left(\zeta_{h}, \zeta_{z}\right)$ is the absolute vorticity vector, $\theta$ is the potential temperature, $\Pi=C_{p}\left(p / p_{\text {ref }}\right)^{\kappa}=C_{p} T / \theta$ is the Exner function, $p$ is the pressure, $p_{\text {ref }}=1000 \mathrm{hPa}$ is a constant reference pressure, $C_{p}$ is the specific heat of air at constant pressure, $\kappa=R / C_{p}, R$ is the gas constant for air, and $T$ is the temperature.

The vorticity equation in flux form (Haynes and McIntyre, 1987 ) is obtained by applying $\hat{\boldsymbol{k}} \cdot \nabla_{h} \times$ to (Eq. 1),

$\frac{\partial \zeta_{z}}{\partial t}+\nabla_{h} \cdot \boldsymbol{Z}+\hat{\boldsymbol{k}} \cdot \nabla_{h} \theta \times \nabla_{h} \Pi=0$,

from which it is clear that $\boldsymbol{Z}$ is the horizontal flux of the vertical component of absolute vorticity, consisting of three parts, $Z_{1}$ the advective flux, $Z_{2}$ the vorticity flux associated with vortex tilting, and $\boldsymbol{Z}_{f}$ the flux of vorticity due to the horizontal force per unit mass $\boldsymbol{F}$ associated with the divergence of the Reynolds stress. This force is generally thought to be due 
primarily to surface friction. The terms $\boldsymbol{Z}_{2}$ and $\boldsymbol{Z}_{f}$ are together called the non-advective flux of vorticity. The vertical component of the baroclinic generation term $\hat{\boldsymbol{k}} \cdot \nabla_{h} \theta \times \nabla_{h} \Pi$ is generally neglected except in highly baroclinic environments such as the hurricane eyewall or mid-latitude fronts.

The divergence equation comes from applying $\nabla_{h}$. to (Eq. 1):

$$
\frac{\partial \delta}{\partial t}+\nabla_{h} \cdot\left[\nabla_{h}\left(v^{2} / 2\right)+\hat{\boldsymbol{k}} \times \boldsymbol{Z}+\theta \boldsymbol{\nabla}_{h} \Pi\right]=0
$$

where $\delta=\nabla_{h} \cdot \boldsymbol{v}_{h}$ is the divergence. The hydrostatic equation in terms of the Exner function is

$\theta \frac{\partial \Pi}{\partial z}+g=0$.

The anelastic mass continuity equation relates the vertical mass flux $M=\rho_{0} v_{z}$ to the horizontal mass divergence $\rho_{0} \delta$

$$
\frac{\partial M}{\partial z}=-\rho_{0} \delta
$$

where the density $\rho_{0}(z)$ is approximated as depending only on height.

The divergence of the advective flux of vorticity $\boldsymbol{Z}_{1}$ can be split into two pieces,

$-\nabla_{h} \cdot Z_{1}=-\boldsymbol{v}_{h} \cdot \nabla_{h} \zeta_{z}-\zeta_{z} \delta$,

the first of which simply advects vertical vorticity in the horizontal plane and the second of which modifies vorticity via vertical stretching. The advection does not change the magnitude of vorticity in a parcel in the flux form of the equations; this role is reserved for the second term, commonly called the stretching term, which can increase or decrease the magnitude of vorticity (but not change the sign). This split is sometimes useful in distinguishing the stretching part of the vorticity flux from the horizontal advection part.

\subsection{Vorticity balance}

We introduce a balance condition which is obtained by dropping the partial time derivative from the vorticity (Eq. 3) equation:

$\nabla_{h} \cdot \boldsymbol{Z}+\hat{\boldsymbol{k}} \cdot \nabla_{h} \theta \times \nabla_{h} \Pi=0$.

As noted above, we can often ignore the vertical component of the baroclinic generation of vorticity, leaving us with the simplified form of vorticity balance,

$\nabla_{h} \cdot \boldsymbol{Z}=0$.

Aside from the neglect of vertical baroclinic generation, this is an exact equation in the steady state.

Vorticity balance is of interest because vorticity imbalance in the sense defined here is an indicator of evolution of the vorticity field. We demonstrate in Sect. 4 that all terms in the vorticity balance equation can be estimated from the data at hand, thus allowing vorticity tendencies to be calculated.
An additional balance condition is obtained by setting the time tendency of divergence to zero in (Eq. 4):

$\nabla_{h} \cdot\left[\nabla_{h}\left(v^{2} / 2\right)+\hat{\boldsymbol{k}} \times \boldsymbol{Z}+\theta \boldsymbol{\nabla}_{h} \Pi\right]=0$.

If the irrotational part of the horizontal velocity as well as the vertical velocity in (Eq. 10) are neglected, then this equation becomes the nonlinear balance equation (Raymond, 1992). Given the velocity and friction fields, this equation can be used in conjunction with the hydrostatic Eq. (5) to diagnose the potential temperature and pressure perturbation fields, or at least that part of those fields not associated with large time tendencies in the divergence. We will not use Eq. (10), but it is included for completeness.

Equations (8-10) are not Galilean-invariant, as the discarded partial time derivative is different in different reference frames. For instance, imagine a disturbance which moves rapidly at constant speed but other than that evolves only slowly with time. In the reference frame moving with the disturbance, the partial derivative of vorticity with time is small. However, this is not true in the stationary frame, since the vorticity at a fixed point in this frame changes rapidly with time as the disturbance passes by. In order to eliminate time tendencies due to system motion, we do all calculations in the co-moving reference frame of the disturbance being studied.

\subsection{Effects of shear on tropical cyclogenesis}

Vertical shear of the horizontal wind has long been considered to be detrimental to both developing and mature tropical cyclones (Gray, 1968, McBride and Zehr, 1981). Considerable attention has been given to the dynamical effects of shear on a strong, isolated vortex (Jones, 1995, 2000a, 2000b; Reasor et al., 2004). In this case one frequently finds a precession of the vortex tilt relative to the shear vector, culminating in some situations in a tilt of the vortex down and to the left of the shear (in the Northern Hemisphere).

In the formation stage of a tropical cyclone the pattern of vorticity is quite different from that seen in a mature cyclone. Most commonly one finds an evolving, chaotic distribution of mesoscale vorticity perturbations produced by convection and other processes, the ensemble of which constitutes the larger pattern of vorticity associated with the parent disturbance, be it a monsoon trough, a tropical wave, or other system. This situation generally has lower Rossby number than the strong vortex case, possibly allowing the use of quasigeostrophic dynamics. Reasor and Montgomery (2001) find a vortex tilt to the left of the shear vector in certain circumstances under these conditions, particularly when a strong negative gradient in potential vorticity exists beyond some radius. However, it is unclear whether the Nuri 1 case falls in this category, and the neglect of the generation of vorticity by convection in that paper may limit its applicability.

We consider here an alternative idealization in which the parent disturbance is assumed to be a vertically aligned 

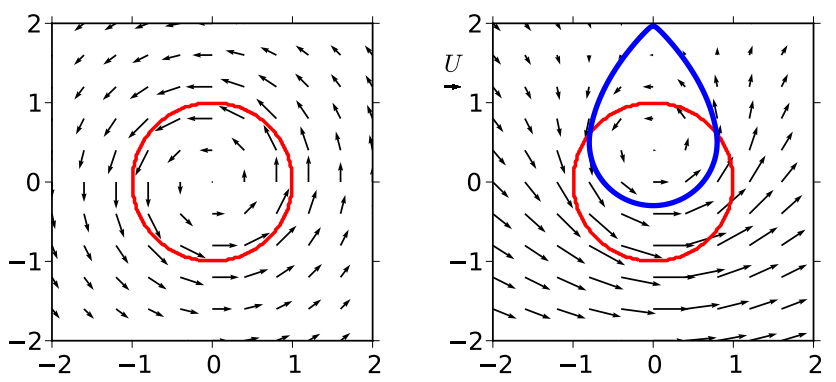

Fig. 1. Schematic illustration of the flow produced by a circular region of constant relative vorticity bounded by the circle (left panel) and the same flow with a superimposed constant wind toward the right (right panel). The constant wind $U$ in the right panel is half that of the induced tangential wind at the radius of the disk of vorticity. This component is graphically represented by the arrow between the plots. The thick line represents the dividing streamline between an interior closed circulation and the exterior flow.

cylinder of enhanced relative vorticity. This vorticity is assumed constant at each level within the cylinder and zero outside. The resulting induced flow is solid body rotation about the cylinder axis within the cylinder and a tangential wind inversely proportional to the distance from the axis outside. We thus neglect the fine-scale structure of the vorticity field. The magnitude of the vorticity and the induced flow can vary with height.

The total flow is the superposition of the flow induced by the vorticity pattern plus the ambient wind. The left panel in Fig. 1 shows schematically the flow induced by the vorticity pattern, which has non-zero vorticity within the confines of the circle. As expected, zero wind is found at the center of the circle. A constant wind toward the right superimposed on this flow produces the flow pattern shown in the right panel of Fig. 1. The point with zero resultant wind has moved away from the center of the disk of vorticity toward the upper boundary, or $90^{\circ}$ to the left of the constant wind. If the constant wind is too strong, then the resultant wind is non-zero everywhere in the flow. This occurs in our simple model when the constant wind exceeds the speed of the induced flow at the edge of the disk of vorticity.

The point of zero wind can be viewed as the center of a closed circulation. In the case of no superimposed constant wind, the radius of the closed circulation is infinite. However, with the superimposed constant flow, the closed circulation occupies a limited area delimited by the thick line in the right panel. The size, shape, and existence of this closed circulation depend on the area and magnitude of the region of non-zero vorticity and on the strength of the constant wind.

For a vertically aligned cylinder of vorticity in shear, the location and extent of the closed region will vary with height and the displacement of the center of the circulation at each level will be normal to the direction of the relative wind at that level. These arguments will be used later to explain the relative locations of circulation centers (in the reference frame of the moving disturbance) at the various stages of developing tropical cyclones. The overlap of closed circulations through a deep layer will be identified as a condition favoring the intensification of a tropical wave or depression.

A vertical cylinder of constant vorticity would of course be subject to disruption by environmental shear, but given the dimensions of a typical wave, the time for this disruption would be long compared to the time scale of the embryonic cyclone embedded in the parent disturbance. Furthermore, the parent disturbance would exhibit its own mechanisms for survival in the face of shear, which would likely include quasi-geostrophic and convective processes. Shear might tilt the cylinder to a certain degree, but if this tilt were weak it would result in only minor complication in the determination of the regions of closed circulation at each level.

If in addition to a solenoidal component to the flow (as represented by the stream function) there were an irrotational component, there would be flow across the streamlines, and parcels could spiral in or out across the boundary between the closed and the external flow. However, if the irrotational part were small compared to the solenoidal part, this would constitute minor leakage between the regions. Time dependence of the flow could also reduce the isolation of the closed region, as noted by Dunkerton et al. (2009).

\subsection{Questions to be addressed}

An analysis of observational data in the light of the vorticity and divergence equations can yield significant insight into the process of tropical cyclogenesis. Among the issues considered here are the following:

1. How does the vertical mass flux profile of convection affect the development of a cyclone? The vorticity Eq. (3) together with Eq. (7) indicate that the strongest spinup exists at levels with the largest values of the product of absolute vorticity and convergence $-\zeta_{z} \delta$. The strongest convergence (strictly, the mass convergence $-\rho_{0} \delta$ ) occurs where the vertical mass flux increases most rapidly with height.

2. What are the effects of shear on the early stages of the development of a cyclone embedded in an extensive region of pre-existing vorticity, such as that provided by a tropical wave? This is a very different situation from that of an isolated vortex in shear, as discussed above.

3. What is the horizontal distribution of vertical stretching responsible for cyclone spinup? Is it concentrated in a few intense VHTs as proposed by Hendricks et al. (2004) and Montgomery et al. (2006), or is it more widely distributed in numerous but less intense convective systems?

4. How close does the pre-cyclone system come to vorticity balance in the boundary layer? 
Table 1. Information about the four flights into Nuri (15-19 August) and one into TCS030 (1-2 September). The second and third columns give the operation altitude of the two aircraft. The fourth column specifies the reference time to which all observations are reduced, given in units of kiloseconds since the UTC beginning of the first date listed in column one. Thus, reference times exceeding $86.4 \mathrm{ks}$ actually occur on the following UTC day. The fifth and sixth columns give the location of the low-level circulation center of the system at the reference time and the eastward and northward components of the observed propagation speed of the system.

\begin{tabular}{cccccc}
\hline Date & P3 & WC130J & Ref time & Ref location & Storm velocity \\
\hline 15-16 August & $2.4 \mathrm{~km}$ & $9.4 \mathrm{~km}$ & $93.0 \mathrm{ks}$ & $\left(145.5^{\circ} \mathrm{E}, 15.5^{\circ} \mathrm{N}\right)$ & $(-4.9,0.0) \mathrm{m} \mathrm{s}^{-1}$ \\
16-17 August & $2.4 \mathrm{~km}$ & $9.4 \mathrm{~km}$ & $86.0 \mathrm{ks}$ & $\left(140.0^{\circ} \mathrm{E}, 16.7^{\circ} \mathrm{N}\right)$ & $(-8.7,0.0) \mathrm{m} \mathrm{s}^{-1}$ \\
17-18 August & $3.6 \mathrm{~km}$ & - & $90.0 \mathrm{ks}$ & $\left(132.7^{\circ} \mathrm{E}, 16.5^{\circ} \mathrm{N}\right)$ & $(-8.7,0.6) \mathrm{m} \mathrm{s}^{-1}$ \\
18-19 August & - & $3.0 \mathrm{~km}$ & $83.0 \mathrm{ks}$ & $\left(127.1^{\circ} \mathrm{E}, 17.1^{\circ} \mathrm{N}\right)$ & $(-6.8,1.9) \mathrm{m} \mathrm{s}^{-1}$ \\
1-2 September & $2.4 \mathrm{~km}$ & $9.4 \mathrm{~km}$ & $76.0 \mathrm{ks}$ & $\left(145.0^{\circ} \mathrm{E}, 14.5^{\circ} \mathrm{N}\right)$ & $(-6.3,0.6) \mathrm{m} \mathrm{s}^{-1}$ \\
\hline
\end{tabular}

\section{Data and methods}

\subsection{TPARC/TCS08}

The TPARC/TCS08 field program took place from 1 August through 3 October 2008. Though the main operations center was located at the US Naval Postgraduate School in Monterey, California, aircraft bases were located in Guam, Taiwan, and Japan. Okinawa was sometimes used as an auxiliary aircraft base. Driftsonde balloons capable of deploying dropsondes from stratospheric elevations, were launched from the Island of Hawaii.

The aircraft available to the project were two WC-130J turboprops from the US Air Force Reserve 53rd Weather Reconnaissance Squadron, the US Naval Research Laboratory (NRL) P-3 aircraft (all based in Guam), the Taiwanese DOTSTAR aircraft, a modified Astra business jet operated by the National Taiwan University (Taipei), and the German Dassault Falcon 20-E5 jet operated by the Deutsches Zentrum für Luft- und Raumfahrt (DLR; Atsugi, Japan). All aircraft were capable of deploying dropsondes. In addition, the DLR Falcon carried downward-looking wind, temperature, and water vapor lidars, while the NRL P-3 carried the National Center for Atmospheric Research's ELDORA radar and a Doppler wind lidar.

\subsection{Observations}

Table 1 gives information about the four aircraft missions into developing tropical cyclone Nuri and the single mission into TCS030. The aircraft involved in the observations of Nuri were the two WC-130Js and the NRL P-3, all operating out of Guam. Though the P-3 generally operated between elevations of $2.4 \mathrm{~km}$ and $3.6 \mathrm{~km}$, it climbed for a short time to $7.3 \mathrm{~km}$ during the first mission in order to deploy dropsondes from a higher altitude. The WC130J deployed a few dropsondes in Nuri from $9.4 \mathrm{~km}$ during the third mission, but had to return to Guam due to mechanical problems. The preferred altitude of operation for the WC130J was $9.4 \mathrm{~km}$, but it descended to $3.0 \mathrm{~km}$ when icing or turbulence became a problem at the higher altitude.

Times are given in UTC in this paper. Local time in Guam is UTC $+10 \mathrm{hr}$. All on-station times for Nuri and TCS030 flights by the P-3 were in daylight. The Nuri flights departed Guam around 08:00 LT and returned around 15:00 LT. The TCS030 flight of the P-3 departed at 05:00 LT and returned near 12:00 LT. The WC-130J generally took off a few hours before the P-3.

The mission of the WC-130Js was to deploy a grid of dropsondes over the disturbance in question from as high an altitude as feasible given the conditions. The main mission of the P-3 was to make Doppler radar measurements of convection using the ELDORA radar. In most cases the strategy was to obtain snapshots of as many convective systems as possible within the cyclone; repeated measurements on convective systems were generally not made, as obtaining a large statistical sample of convection was considered to be more valuable than following the life cycles of a few systems. In addition, the P-3 deployed dropsondes along the flight path and made Doppler wind lidar measurements of the atmospheric boundary layer beneath the aircraft.

The ELDORA radar was configured as shown in Table 2. The unambiguous range of $75 \mathrm{~km}$ means that Doppler radar measurements in a $150 \mathrm{~km}$ swath were made, centered on the P-3 track, albeit with reduced spatial resolution and sensitivity at the outer limits. Since ELDORA is an X-band radar, attenuation is a significant issue. However, the large unambiguous velocity of $62 \mathrm{~m} \mathrm{~s}^{-1}$ means that unfolding of radial velocities is not a significant problem with the systems observed in this case study.

The Doppler radar measurements from the P-3 aircraft and dropsondes from the P-3 and the WC130J were used for the first three Nuri missions to produce gridded horizontal and vertical winds satisfying mass continuity using a three-dimensional variational analysis scheme (3D-VAR). This scheme is described in detail by López Carrillo and Raymond (2010). Only dropsondes deployed during the 4-6 hr in which the P-3 was flying in the system were used. The 
Table 2. Characteristics of the ELDORA radar during TPARC/ TCS08.

\begin{tabular}{ll}
\hline Radar characteristic & Value \\
\hline Wavelength & $3.2 \mathrm{~cm}$ \\
Beamwidth $(\mathrm{H} \mathrm{x} \mathrm{V)}$ & $1.8^{\circ} \times 2.0^{\circ}$ \\
Antenna gain & $39.2 \mathrm{~dB}$ \\
Beam tilt angle & $+15.6^{\circ}$ fore; $-16.5^{\circ} \mathrm{aft}$ \\
Antenna rotation rate & $\approx 78^{\circ} \mathrm{s}^{-1}$ \\
Peak transmitted power & $40 \mathrm{~kW}$ \\
Pulse repetition frequency & $1600 / 2000 \mathrm{~Hz}$ \\
Minimum detectable signal at $10 \mathrm{~km}$ & $-12 \mathrm{dBZ}$ \\
Unambiguous range & $75 \mathrm{~km}$ \\
Unambiguous velocity (dual PRT) & $\pm 62 \mathrm{~m} \mathrm{~s}$ \\
Number of frequencies & 3 \\
Total cell length & $150 \mathrm{~m}$ \\
Along track sweep spacing & $\approx 500 \mathrm{~m}$ \\
\hline
\end{tabular}

fourth Nuri mission produced only dropsonde data from the WC-130J, and these soundings (deployed from $3 \mathrm{~km}$ for operational reasons) were used to produce an analysis with the 3D-VAR system.

For the first three Nuri missions for which radar data are available and for the single TCSO30 mission, the grid size is $0.125^{\circ}$ in both latitude and longitude (roughly $14 \mathrm{~km}$ ) and $0.625 \mathrm{~km}$ in the vertical. The gridded domain is $5^{\circ} \times 5^{\circ} \times$ $20 \mathrm{~km}$ or $40 \times 40 \times 32$ cells for Nuri missions 2 and 3 and $7^{\circ} \times$ $7^{\circ} \times 20 \mathrm{~km}$ or $56 \times 56 \times 32$ cells for Nuri mission 1 and the TCS030 mission. For the fourth Nuri mission in which only dropsonde data are available, the horizontal grid resolution is also $0.125^{\circ}$ with a $4^{\circ} \times 4^{\circ}$ domain size, for a grid with $32 \times 32 \times 32$ cells.

Though the 3D-VAR scheme produces results in the entire domain analyzed, these results are not reliable outside of the region where data exist. The results are therefore masked to include only regions which contain either radar or dropsonde data (or both). At higher altitudes radar data cover less area. Furthermore, the deepest dropsonde soundings begin near roughly $9 \mathrm{~km}$. For this reason, the regions above $12 \mathrm{~km}$ are ignored and most results come from observations at $5 \mathrm{~km}$ and below. The 3D-VAR scheme is capable of incorporating high elevation angle radar data. However, radar rays with elevation angles exceeding $\pm 30^{\circ}$ were not used in the analysis in order to avoid contaminating our results with uncertainties in the estimation of particle terminal velocities. Results obtained with and without this limitation are quite similar, but the high elevation angle data introduce some "cosmetic" artifacts in vertical velocities which we prefer to avoid. A minimum of 200 radar radial velocity samples are required in each grid cell and the condition $a_{2} \geq 0.03$ (a measure of the quality of the geometry for dual Doppler analysis) is imposed in order to ensure that only valid dual Doppler results are included (see López and Raymond 2010). These condi- tions largely eliminate random outliers in the radar synthesis without significantly limiting radar coverage.

Before applying the 3D-VAR scheme, radar and dropsonde observations are adjusted to their corresponding positions at a specified reference time using the observed propagation velocity of the disturbance over the measurement period. System positions were estimated from vorticity fields in National Centers for Environmental Prediction Final Analysis (NCEP FNL) data. Both propagation velocities and reference times are listed in Table 1. The low-level circulation centers at the reference time for each mission are also given in this table.

Different ways of estimating system propagation speeds can yield somewhat different answers early in a system's development. In particular, Montgomery et al. (2010) estimate that Nuri was moving to the west approximately $2 \mathrm{~m} \mathrm{~s}^{-1}$ faster than we estimated for the first observational period. Tests indicate that our results are not overly sensitive to such differences.

\subsection{Vorticity analysis}

Once the velocity grid is obtained, all components of the vorticity are computed using centered differences. The first two terms on the right side of (Eq. 3) are then computed from the vorticity and the velocity. The advection and stretching components of the first term are also computed separately as indicated in (Eq. 7).

In order to compute the third term, the surface wind stress needs to be calculated and an assumption has to be made about the depth over which the stress is distributed. The surface stress $\tau$ is computed from a bulk flux formula

$\boldsymbol{\tau}=-\rho_{\mathrm{BL}} C_{D}\left|\boldsymbol{U}_{\mathrm{BL}}\right| \boldsymbol{U}_{\mathrm{BL}}$

where a subscript BL indicates a boundary layer value, with $\rho$ indicating air density and $\boldsymbol{U}$ indicating the horizontal wind in the earth's reference frame. Since Doppler radar observations close to the surface are problematic due to sea clutter, $\boldsymbol{U}_{\mathrm{BL}}$ is derived primarily from radar (and dropsonde) winds at the first analyzed level above the surface at $z=0.625 \mathrm{~km}$.

Results from the CBLAST (Coupled Boundary Layer Air-Sea Transfer) experiment (figure 5 of Black et al., 2007) suggest an estimate for the drag coefficient

$C_{D} \approx\left(1+0.028\left|\boldsymbol{U}_{\mathrm{BL}}\right|\right) \times 10^{-3}$

thought to be valid up to approximately $30 \mathrm{~m} \mathrm{~s}^{-1}$. Since CBLAST results are for $10 \mathrm{~m}$ winds, our use of winds near $625 \mathrm{~m}$ results in a slight overestimate for the surface stress.

The specific frictional force $\boldsymbol{F}$ is postulated to take the form

$\rho \boldsymbol{F} \approx \boldsymbol{\tau} \exp \left(-z / z_{s}\right) / z_{s}$

where a scale height of $z_{s}=1.25 \mathrm{~km}$ is chosen to represent the average depth of the planetary boundary layer (PBL) in 


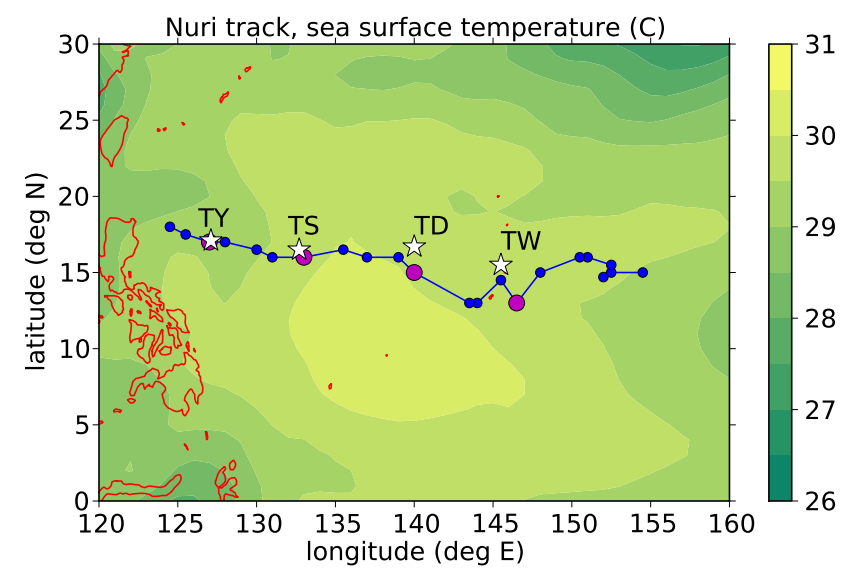

Fig. 2. Sea surface temperature and the observed low-level circulation centers of Nuri (white stars) during tropical wave (TW), tropical depression (TD), tropical storm (TS), and typhoon (TY) missions. The blue line indicates the track of the vorticity center in $850 \mathrm{hPa}$ FNL data, with the blue dots spaced at $6 \mathrm{hr}$ intervals. The larger magenta dots indicate the 00:00 UTC FNL positions nearest to the reference times shown in Table 1 for the observational missions.

tropical regions. The postulated scale height is consistent with the idea that surface friction is mixed through the full PBL, i.e., the layer containing boundary layer clouds as well as the sub-cloud layer, via turbulent eddies. Unfortunately, not enough is known about boundary layers topped by convective clouds in developing tropical storms to justify a more refined estimate of the vertical distribution of the drag force resulting from the surface stress. Given these uncertainties, $\boldsymbol{F}$ is probably known to within only a factor of two. However, this accuracy is sufficient to draw some significant conclusions, as noted below.

With $\boldsymbol{F}$ roughly known, the third term on the right side of (Eq. 2) is estimated. Substituting this equation in (Eq. 3), dropping the vertical baroclinic term, integrating over a horizontal area $A$, and applying Gauss's law, we obtain the tendency of the absolute circulation around the area $A$,

$\frac{d \Gamma}{d t}=-\oint v_{n} \zeta_{z} d l+\oint \zeta_{n} v_{z} d l+\oint F_{t} d l$,

where the line integrals are taken to be in the counterclockwise direction over the periphery of $A, v_{n}$ and $\zeta_{n}$ are the horizontal outward normal components of the velocity and vorticity, and $F_{t}$ is the component of $\boldsymbol{F}$ tangential to the periphery of $A$ in the direction of the integration. Thus, the circulation around the area $A$ depends only on what is happening on the periphery of $A$ and not in the interior. The first term on the right is the spinup tendency due to the convergence of absolute vorticity, the second expresses the effect of vortex tilting on the periphery of $A$, and the third is the spindown tendency due to friction.

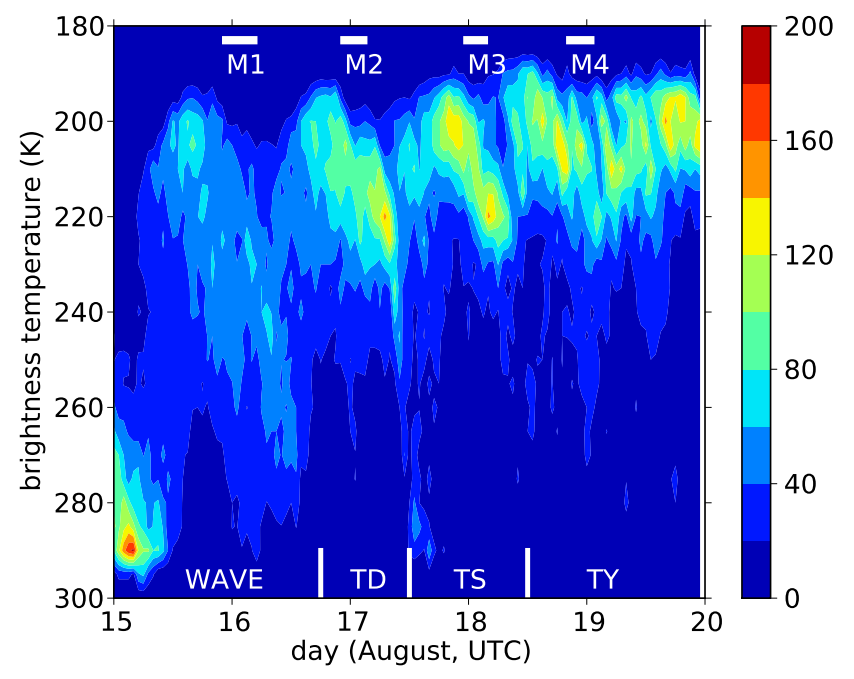

Fig. 3. Histogram of satellite infrared brightness temperatures of pixels in a $5^{\circ} \times 5^{\circ}$ square as a function of time, centered on tropical cyclone Nuri as it intensified. The stages of the storm (wave, tropical depression, tropical storm, typhoon) are shown, as well as the approximate times of the four aircraft missions, M1-M4 (see Table 1). The color scale indicates the number of pixels with the given temperature at the given time.

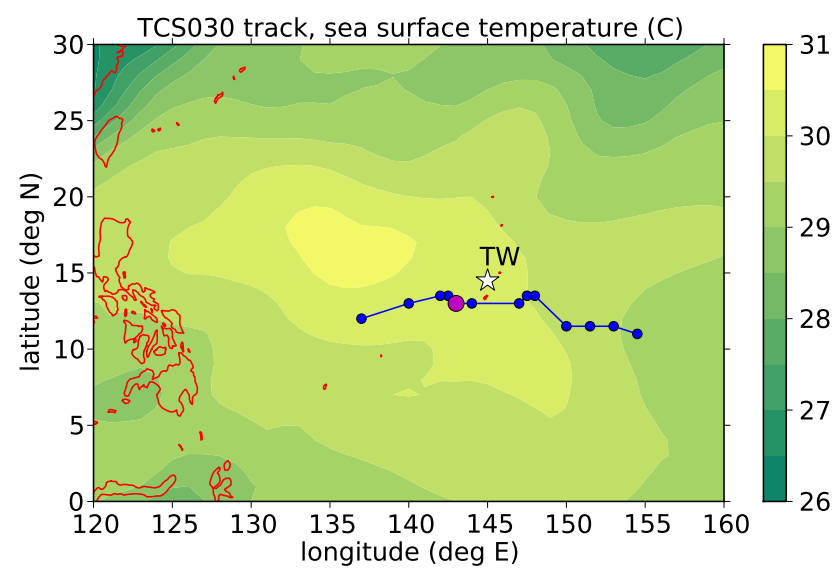

Fig. 4. As in Fig. 1 except for tropical wave TCS030.

The terms on the right side of (Eq. 14) may be computed directly, or by area integrating the right side of (Eq. 3). We choose the latter approach as it is computationally simpler. In addition, by expressing the horizontal velocity $\boldsymbol{v}_{h}$ as relative to the motion of a propagating system, the circulation tendency around the moving system can be computed using (Eq. 14). 


\section{Results}

In this section we document the overall development of Nuri and of the tropical wave TCS030. We then examine the vorticity structure of these systems and finally analyze the vorticity dynamics of intensification.

\subsection{Overview of development}

Figure 2 shows the locations of tropical cyclone Nuri determined from FNL $850 \mathrm{hPa}$ vorticity patterns as well as low-level circulation centers from our observational analysis, along with the Reynolds (Reynolds and Marsico, 1993) sea surface temperature (SST) distribution for the period of Nuri's intensification. Previous to the typhoon stage, Nuri passed over SSTs of approximately $30^{\circ} \mathrm{C}$. It later encountered decreasing SSTs. The FNL positions are obtained from subjective estimates of the vorticity center. As the scatter in successive position estimates shows, this procedure is not very precise in the tropical wave stage. It also appears to lead to a systematic southward displacement of the estimated position of Nuri during this phase, compared to our observations of the low-level circulation center.

Figure 3 shows a time series of the distribution in satellite infrared brightness temperature in a $5^{\circ} \times 5^{\circ}$ square centered on Nuri as it intensified and moved to the west. The input for this figure is a series of Japanese MTSAT geosynchronous satellite infrared images interpolated to a longitude-latitude grid with a resolution of $0.2^{\circ} \times 0.2^{\circ}$. A pronounced diurnal cycle is seen, with coldest cloud tops occurring near 18:00 UTC. This diurnal cycle diminishes in amplitude as the storm intensifies and produces a more extensive region of high overcast. Note that the aircraft missions took place during periods of warming cloud tops. Operational constraints prevented us from exploring the diurnal cycle with the aircraft.

Figure 4 shows the SST at the time TCS030 was observed and its location at this time. SSTs were in excess of $30^{\circ} \mathrm{C}$ and the system was moving toward even warmer water. In spite of these favorable conditions, TCS030 did not develop. Instead it fluctuated in intensity and finally made landfall in the southern Philippines as a tropical wave. The white star in Fig. 4 is located near the most intense convection observed in TCS030 since no low-level circulation center was obvious. This estimate is displaced well to the northeast of the position estimate based on FNL $850 \mathrm{hPa}$ vorticity.

\subsection{Vorticity structure}

Figures 5-7 show the storm-relative winds and absolute vorticity at $1.2 \mathrm{~km}$ and $5 \mathrm{~km}$ for the first three missions into tropical cyclone Nuri. The track of the P-3 aircraft and locations of usable dropsondes are shown in the left-hand panels. Reflectivities greater than $25 \mathrm{dBZ}$ at $5 \mathrm{~km}$ are shown as grayscale insets in the right-hand panels.
As storm-relative winds are used, the circulation centers in both panels are physically significant since the associated streamlines are close to being parcel trajectories. These circulation centers are also important thermodynamically, as they are protected from the injection of dry air from outside the system (Dunkerton et al., 2009). The $1.2 \mathrm{~km}$ circulation centers are listed in Table 1 and shown in Fig. 5.

The low-level circulation center is somewhat ill-defined in the case of Nuri 1 (first Nuri flight), though it is plausibly located at the white star in Fig. 5. The low-level vorticity in Nuri 1 shows a northwest-southeast band to the northeast of the assumed center (Fig. 5) and an otherwise random pattern of vorticity fluctuations. The band bounds the southern limit of the strong cyclonic flow on the north side of the system. At $5 \mathrm{~km}$, the pattern is quite different, with the strongest vorticity on the east and southeast sides of the disturbance, encompassing the $5 \mathrm{~km}$ circulation center. Visual observation from the P-3 indicated that this region of vorticity aloft coincided with a large stratiform rain area. This is in contrast with the western part of the disturbance, which exhibited a much more convective structure. The elongated center of the system-relative circulation at $5 \mathrm{~km}$ is in the form of a northeast-southwest band displaced $\approx 3^{\circ}$ to the southeast of the low-level center. The southwestern end of this band is close to the position inferred from FNL $850 \mathrm{hPa}$ vorticity. Maximum relative winds are near $10 \mathrm{~m} \mathrm{~s}^{-1}$ at both levels.

Given the uncertainty in the Nuri 1 propagation velocity, Fig. 5 was replotted (not shown) with an assumed propagation velocity of $(-7,0) \mathrm{m} \mathrm{s}^{-1}$, which is about $2 \mathrm{~m} \mathrm{~s}^{-1}$ faster to the west than indicated in Table 1 and closer to the observed propagation speed for Nuri 2 and Nuri 3. This change in the propagation velocity moves the circulation centers at both levels to the north-northeast by about $0.6^{\circ}$, but does not change the character of the overall storm-relative flow or any of the conclusions drawn from this plot.

The strength of vorticity perturbations has intensified at both $1.2 \mathrm{~km}$ and $5 \mathrm{~km}$ in Nuri 2 (second Nuri flight; see Fig. 6), though there is still little system-wide organization at $1.2 \mathrm{~km}$. The $1.2 \mathrm{~km}$ circulation center is located at the northwest corner of the observed region in this case and the circulation center at $5 \mathrm{~km}$ is displaced $\approx 2^{\circ}$ to the south-southeast of the low-level center. At $5 \mathrm{~km}$ there is a rather strong north-south band of vorticity located roughly between the low and mid-level circulation centers. The overall circulation is slightly stronger than that seen in Nuri 1 . Maximum relative winds are near $15 \mathrm{~m} \mathrm{~s}^{-1}$, with somewhat stronger winds occurring at $5 \mathrm{~km}$.

Figure 7 shows that the circulation pattern has changed drastically for Nuri 3 (third Nuri flight), with a strong central vorticity maximum at $1.2 \mathrm{~km}$ and a spiral band of vorticity linked to the central maximum. A nearly co-located maximum in vorticity exists at $5 \mathrm{~km}$. The maximum winds are about $20 \mathrm{~m} \mathrm{~s}^{-1}$ at both levels, with the largest values occurring near the central vorticity maximum. 
Nuri 1: relative winds, absolute vorticity $\left(\mathrm{ks}^{-1}\right)$

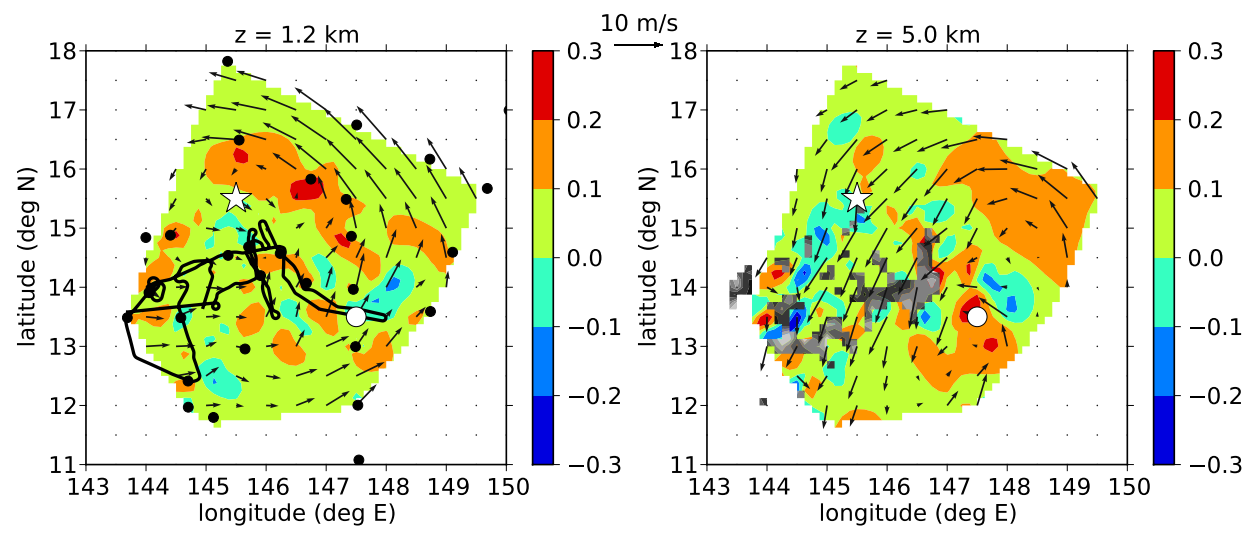

Fig. 5. Absolute vorticity (color levels) and storm-relative winds for Nuri mission 1 at $1.2 \mathrm{~km}$ (left panel) and $5 \mathrm{~km}$ (right panel). The black line and black dots in the left panel indicate the P-3 aircraft track and the locations of P-3 and WC-130J dropsondes. The gray scale insets in the right panel show regions of radar reflectivity exceeding $25 \mathrm{dBZ}$ at $5 \mathrm{~km}$. The white star and the white circle indicate the system-relative circulation centers in the PBL and at $5 \mathrm{~km}$ respectively. The white areas reflect the geometry of the mask chosen for this mission.

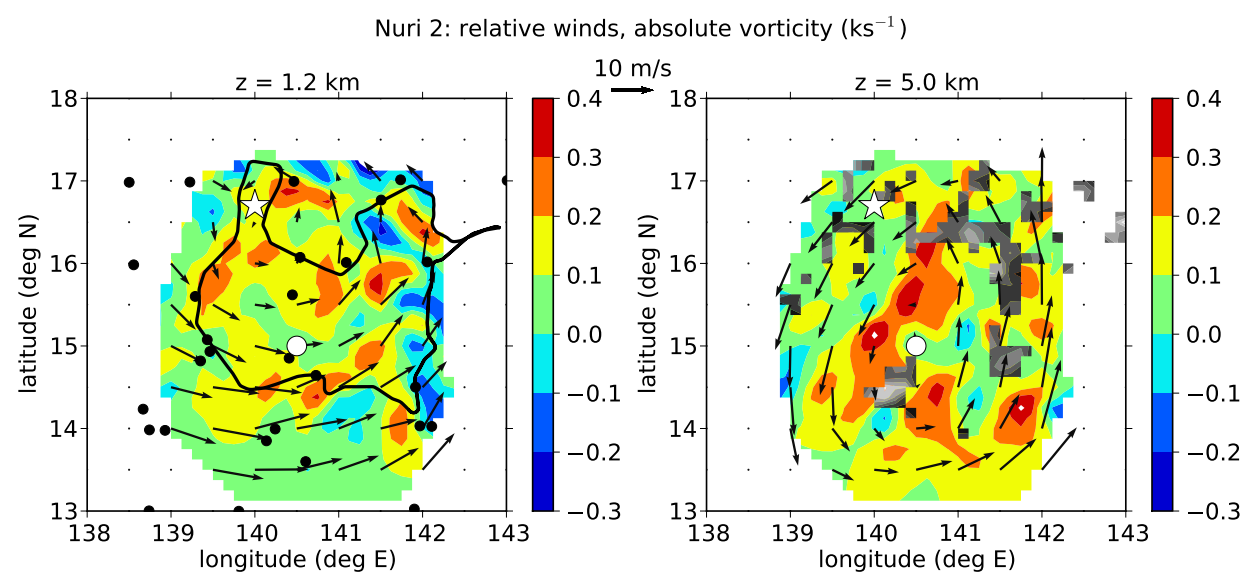

Fig. 6. As in Fig. 5 except second Nuri mission.

Nuri 3: relative winds, absolute vorticity $\left(\mathrm{ks}^{-1}\right)$

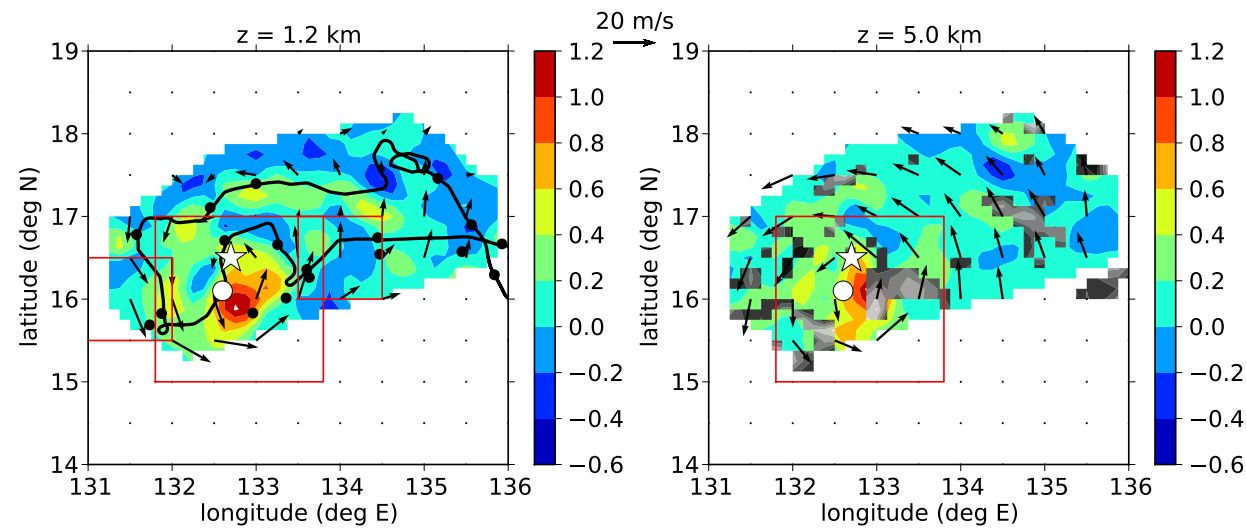

Fig. 7. As in Fig. 5 except third Nuri mission. The large red box indicates the central core region and the two smaller red boxes on opposite sides of the core are averaging regions for the wind profile calculation. 


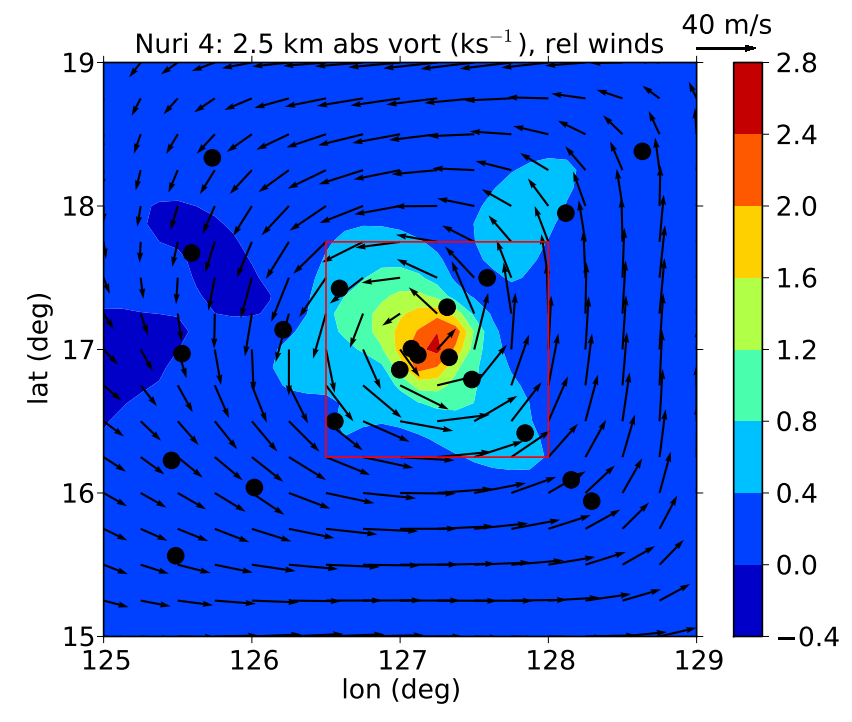

Fig. 8. Vorticity and storm relative winds at $2.5 \mathrm{~km}$ for the fourth Nuri mission (WC-130J dropsondes only). The black dots indicate dropsondes of sufficient quality to be used. The red box indicates the central core region.

Figure 8 shows the vorticity and storm-relative winds for mission 4. No radar reflectivities are available due to the absence of the P-3 in this mission. The circulation center is clearly defined at this stage. Due to the lack of observations between the legs of the "X" pattern flown by the WC-130J, the analyzed winds and vorticity in the outer regions are distorted. However, with the concentration of observations near the circulation center, the winds are likely to be more reliable there. Maximum winds are near $40 \mathrm{~m} \mathrm{~s}^{-1}$ and the near-core circulation is highly symmetric.

The circulation in the case of TCS030 (Fig. 9) is very weak, with maximum system-relative winds less than $10 \mathrm{~m} \mathrm{~s}^{-1}$. To the extent that a circulation can be inferred at $1.2 \mathrm{~km}$, it is centered at the far northeast corner of the observed region. The system-relative flow through the system at low levels is from the west. Though there is a region of moderately strong $5 \mathrm{~km}$ vorticity near the northeast corner, the flow at this level does not suggest a closed circulation even in storm-relative coordinates. In general, vorticities are less than they are in Nuri 1.

Figure 10 shows histograms of the relative frequency of occurrence of vorticity values at $1.2 \mathrm{~km}$ elevation seen in Figs. 5-7 and 9. (Results from Fig. 8 are not included, as fine resolution radar data were not available in this case.) As Nuri intensified, larger values of vorticity developed as expected. However, the overall distribution broadened as well, with more negative values also occurring. Negative absolute vorticity values are likely produced by the tilting term in the vorticity equation; the only other possible mechanism would be convergence of pre-existing negative vorticity, which could in principle occur, but seems less likely. Increased convec- tive activity in shear, which promotes tilting, is probably how this happens. The strong vorticities in the central maximum seen in Fig. 7 are highly limited in areal coverage and appear in the extreme tail of the Nuri 3 vorticity distribution. The distribution of vorticity in the tropical wave TCS030 is somewhat similar to that in the tropical wave stage of Nuri, but with more negative and fewer positive values of absolute vorticity.

Figure 11 shows vertical profiles of wind for the Nuri missions and the TCS030 mission. All but the Nuri 3 profiles were obtained from averaging analyzed winds from the 3DVAR scheme over the full domains of Figs. 5, 6, 8, and 9. There is likely to be some contamination of the environmental flow with storm generated winds, but by averaging over the entire domain, which is at least somewhat centered on the disturbance, much of this contamination should average out. For Nuri 3, one-degree-square regions on opposite sides of the central core (see Fig. 7) rather than the entire domain are averaged in order to cancel out axisymmetric storm perturbations to the flow. This seems preferable to averaging over the full observed domain, since the concentration of observations on the north side of the core of Nuri 3 is likely to increase greatly the contamination of the environmental flow with cyclone-generated winds.

Figure 11 shows moderately strong shears of $\approx 7 \mathrm{~m} \mathrm{~s}^{-1}$ between the surface and $6 \mathrm{~km}$ in Nuri 1 and Nuri 2. The Nuri 3 shear is considerably stronger, of order $15 \mathrm{~m} \mathrm{~s}^{-1}$. The shear was strong enough in the real time forecast for TPARC/TCS-08 forecasters to discount the possibility that Nuri would intensify. The initial mission was therefore undertaken as a probable "null" case. The relative wind profile in TCS030 differed little in essential characteristics from that seen in Nuri 1 with the exception that strong northerly system-relative flow existed at middle and upper levels.

\subsection{Circulation dynamics}

As noted in Sect. 3, the advective ( $\boldsymbol{Z}_{1}$ in (Eq. 3)) and nonadvective fluxes $\left(\boldsymbol{Z}_{2}+\boldsymbol{Z}_{f}\right)$ of the vertical component of absolute vorticity are estimated from the velocity and vorticity fields. Figures $12-15$ show the total vorticity flux and the vorticity tendency due to vertical stretching for Nuri 1-3 and TCS030 in the PBL and at $5 \mathrm{~km}$. Note that the scales for the vorticity flux and the stretching tendency differ between figures. The stretching tendency $-\zeta_{z} \nabla_{h} \cdot \boldsymbol{v}_{h}$ is shown rather than the total tendency because stretching is the main mechanism by which parcel values of vorticity are increased, at least in regions of small tilting tendency. The vorticity advective tendency $-\boldsymbol{v}_{h} \cdot \nabla_{h} \zeta_{z}$ exhibits complex patterns which are irrelevant to the parcel increase in vorticity since advection simply moves parcels around without changing their vorticity. Of course the advective contributions are needed to compute the overall circulation tendency given by (Eq. 14) since they can move parcels in and out of the circulation domain. 


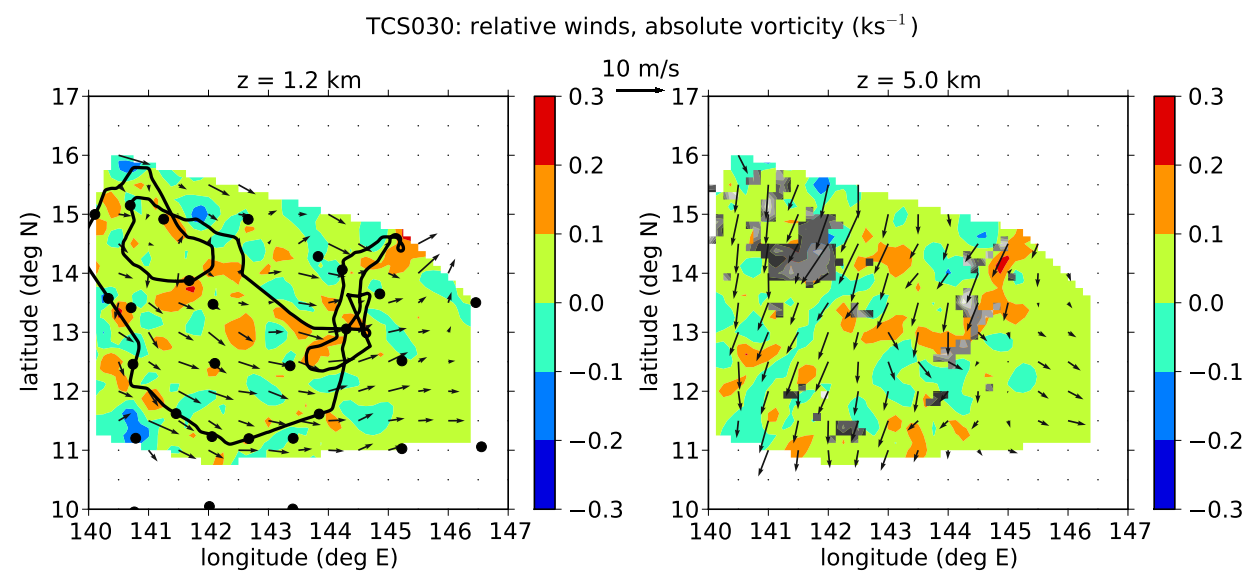

Fig. 9. As in Fig. 5 except the TCS030 mission. No circulation centers are indicated.

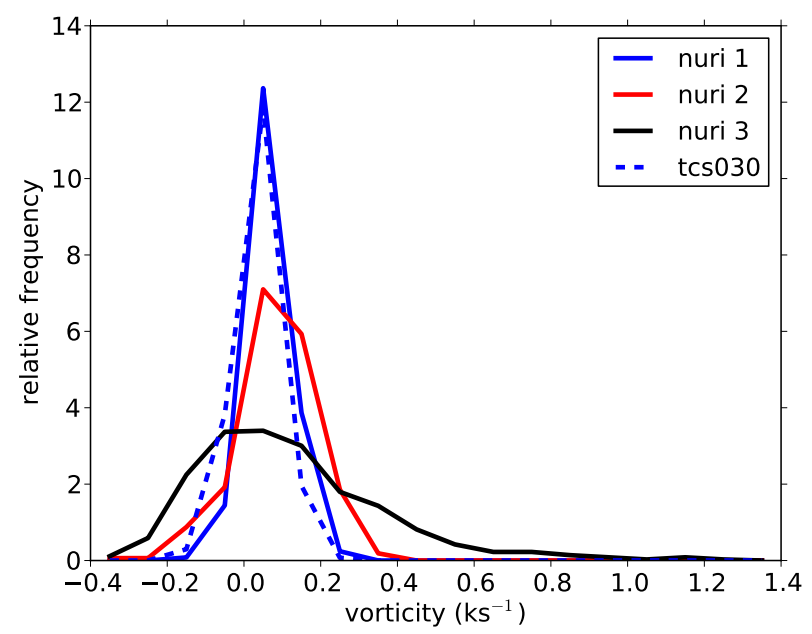

Fig. 10. Relative frequency of absolute vorticity values at $1.2 \mathrm{~km}$ in Figs. 5-7 and 9.

Also shown is the total vorticity flux. The vorticity flux (but not the stretching tendency) has been low-pass filtered with a filter length of $0.5^{\circ}$ to improve the clarity of the overall vorticity flow patterns. Mostly the vorticity flux reflects the horizontal velocity field, demonstrating the large magnitude of the advective part of the vorticity flux relative to the nonadvective part.

The stretching tendency of vorticity indicates regions of mass convergence, so maxima in this quantity indicate regions of convection with significant convergence in the boundary layer. Comparison of stretching maxima in the PBL with regions of strong $5 \mathrm{~km}$ reflectivity in the right panels of Figs. 5-7 and 9 shows moderately good, but not perfect agreement in this respect. Notable examples exhibiting strong correlations in this regard include the convection near $\left(145.8^{\circ} \mathrm{E}, 14.2^{\circ} \mathrm{N}\right)$ in Nuri $1,\left(140.2^{\circ} \mathrm{E}, 14.4^{\circ} \mathrm{N}\right)$ in Nuri 2, and $\left(133.0^{\circ} \mathrm{E}, 16.0^{\circ} \mathrm{N}\right)$ in Nuri 3. The strongest stretching tendencies at $5 \mathrm{~km}$ are greater than PBL tendencies in Nuri 1, Nuri 2, and in TCS030.
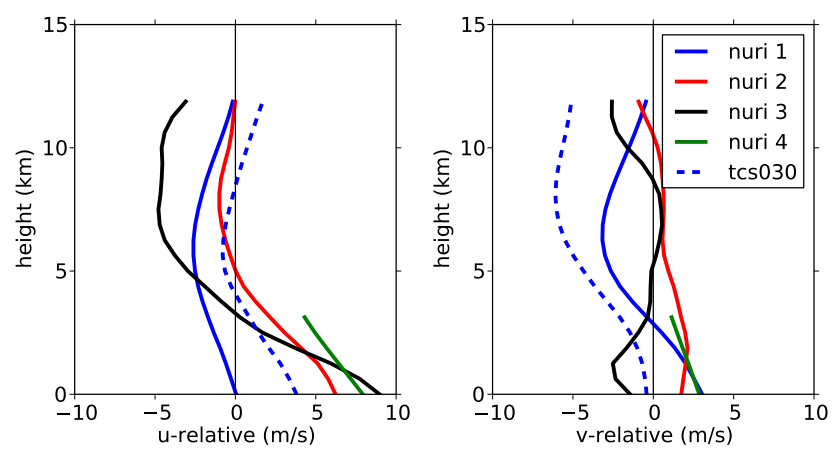

Fig. 11. Storm-relative westerly (left panel) and southerly (right panel) wind components for the four Nuri missions and TCS030.

As Nuri intensifies, the regions of stretching become fewer and more intense, culminating in a single strong central vortex in Nuri 3. This suggests a transformation from scattered ordinary convection to a more limited number of strong convective systems reminiscent of VHTs, which in turn give way to the cyclone eyewall. Such behavior is seen in the threedimensional numerical simulations of Nguyen et al. (2008).

Circulations of vorticity flux in the PBL in Nuri 1 and 2, as seen in Figs. 12-14 appear to be closed, but the observed regions are too restricted to say with absolute certainty. At $5 \mathrm{~km}$ the Nuri 1 circulation also suggests closure. For Nuri 2 at $5 \mathrm{~km}$ and Nuri 3 at both altitudes the vorticity flux circulation is clearly closed. These results suggest that regions of strong vorticity created by stretching are not exported from Nuri during its growing stage. The pattern of vorticity flux in TCS030 in the PBL may be closed, but observations do not extend far enough to the north to verify this. At $5 \mathrm{~km}$ there is no hint of a closed circulation in TCS030, allowing export of generated vorticity at this altitude.

The centers of vorticity circulations are near, but not necessarily colocated with the centers of mass circulations. This difference in location is due to a combination of 


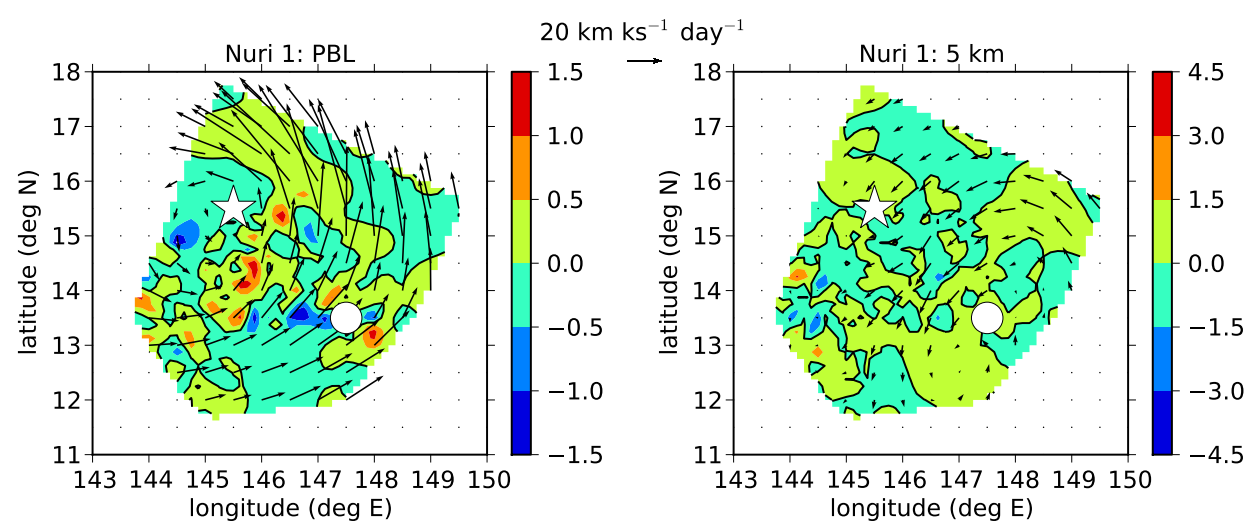

Fig. 12. Stretching tendency (filled contours) for vorticity and total vorticity flux (arrows) in the PBL $(0<z<1.2 \mathrm{~km}$; left panel) and at $5 \mathrm{~km}$ (right panel) as calculated from the analyzed wind fields for Nuri 1. The white stars (PBL) and circles (5 km) indicate the PBL storm-relative circulation center and the black contours indicate zero stretching tendency. The vorticity flux has been low-pass filtered with a filter length of $0.5^{\circ}$ for clarity of the overall pattern, but the stretching tendency has not.
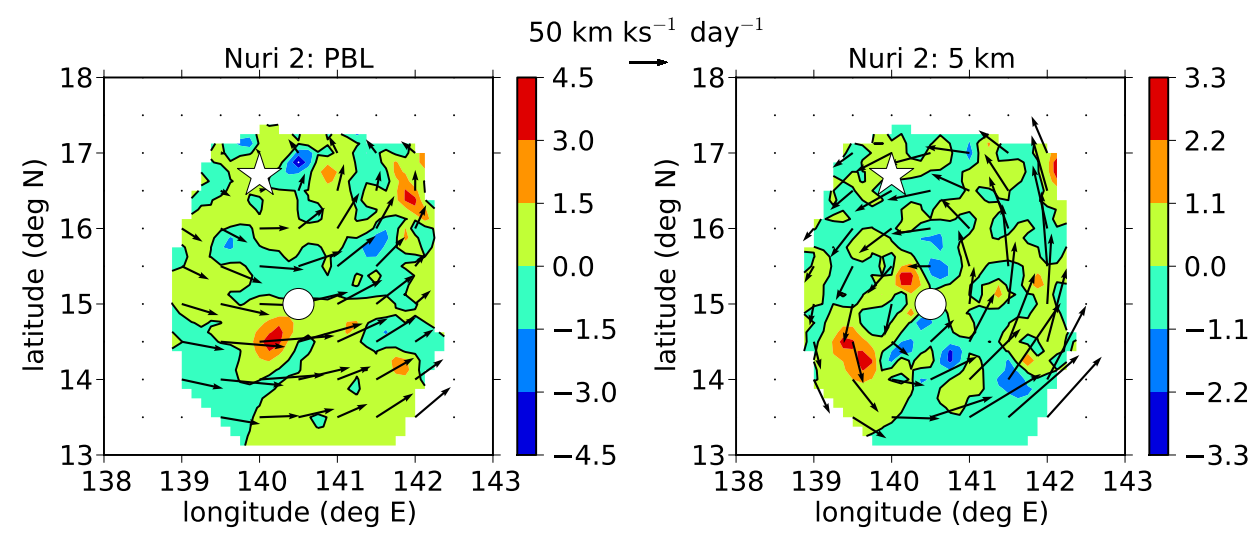

Fig. 13. As in Fig. 12 except for Nuri 2. Note the change in scales.

non-advective fluxes, i.e., tilting and frictional fluxes represented by the second and third terms on the right side of (Eq. 3).

Figures 16-22 show vertical profiles of planetary and absolute circulation $\Gamma$ around the observed regions in Figs. 58 and 9 as well as the integrated vertical mass flux $M$. In addition, the vertical profiles of the various components of circulation tendency are shown. By hypothesis, the friction tendency is concentrated primarily in the PBL. The domain sizes are different for each case. However, the magnitude of the integrated planetary circulation, which is roughly the Coriolis parameter times the area, gives a visual estimate of the relative domain sizes between plots since the Coriolis parameter is very similar in all cases.

Figure 16 shows profiles for Nuri 1. The maximum circulation in this tropical wave case occurs in a nearly uniform layer from the surface up to $4 \mathrm{~km}$ with a value of roughly twice the planetary circulation. The circulation decreases monotonically above this level. The vertical mass flux maximizes at high levels, near $10 \mathrm{~km}$ elevation and is actually negative at low levels, suggesting that downdrafts are strong there. The vertical derivative of mass flux is very small or negative in the PBL. Thus, boundary layer convergence associated with convection is small and the circulation tendency due to vorticity convergence near the surface is correspondingly small. The frictional spindown tendency exceeds vorticity convergence below $1 \mathrm{~km}$. Vorticity convergence increases up to the mid-troposphere and then decreases. Above this level, tilting makes a significant positive contribution to the circulation tendency.

Nuri 2 (Fig. 17) exhibits a very different pattern, with the vorticity convergence term greatly exceeding frictional spindown in the PBL. This is related to the rapid increase in vertical mass flux with height and the associated strong mass convergence in the PBL. The vertical mass flux maximizes near an elevation of $5 \mathrm{~km}$, which is significantly lower than in Nuri 1. The contribution of tilting is comparatively weak at all levels. The surface circulation is still only about twice the planetary circulation, but the maximum circulation has increased to three times the planetary circulation near $5 \mathrm{~km}$. 


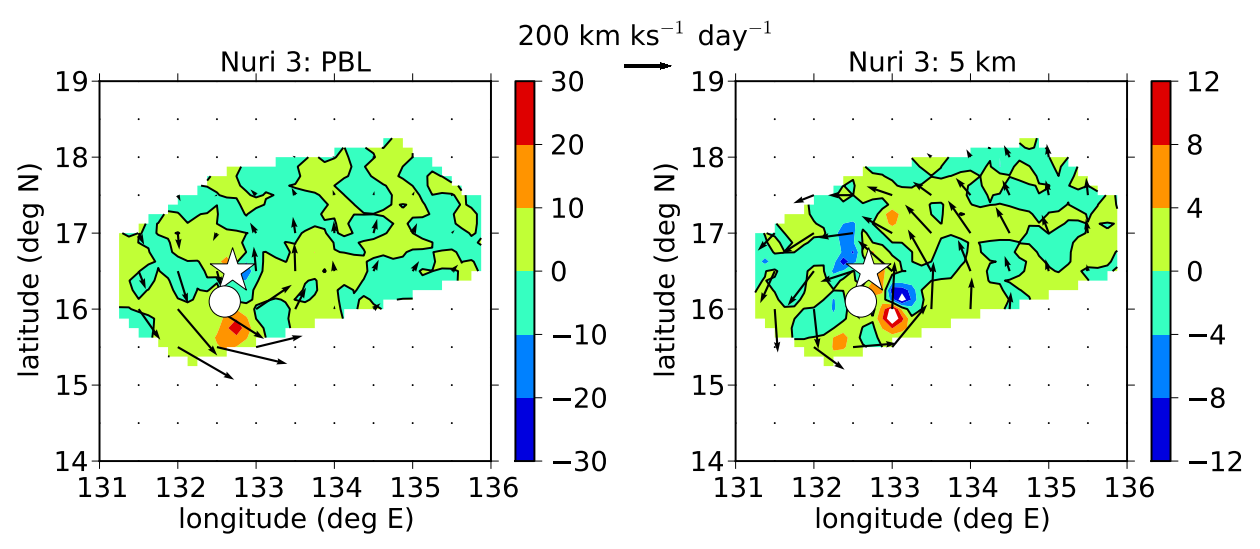

Fig. 14. As in Fig. 12 except for Nuri 3. Note the change in scales.
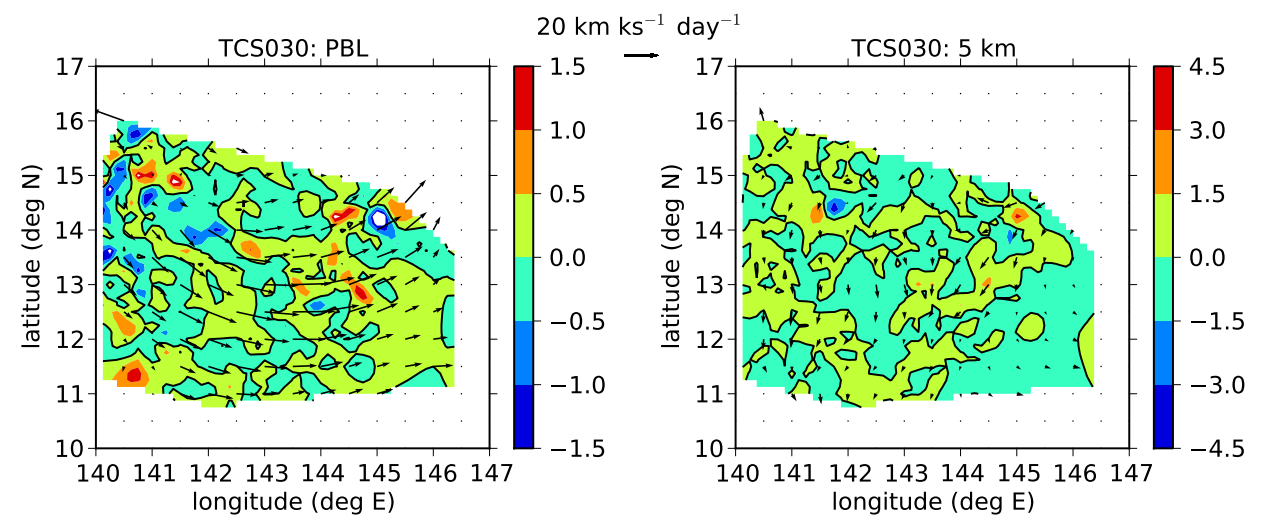

Fig. 15. As in Fig. 12 except for TCS030. No circulation centers are shown in this case.

Figure 18 shows that for Nuri 3 the circulation measured relative to the planetary circulation has increased at all levels in comparison to Nuri 2. The elevation of maximum vertical mass flux has increased to near $6 \mathrm{~km}$ and the mass convergence in the PBL is less than for Nuri 2. Tilting contributes negatively to the circulation tendency in the lower troposphere and positively in the upper troposphere.

The circulation tendency due to vorticity convergence is actually negative in the PBL. This is due to the apparent suppression of stretching away from the central vorticity maximum. The circulation tendency in the PBL for a $2^{\circ}$-square box centered on the core (Fig. 19) is slightly positive, but is indistinguishable from zero given the potential errors in the calculation of surface friction. Comparison of Fig. 18 and 19 also shows that the region within the $2^{\circ}$ box is responsible for $2 / 3$ of the mass flux and circulation and nearly $100 \%$ of the circulation at the surface.

Figures 20 and 21 show the corresponding patterns for Nuri 4 except that the region over which the integration is done for 21 is the $1.5^{\circ}$ box illustrated in Fig. 8. As for Nuri 3 , the circulation tendency due to convergence over the full domain is small, with a net spindown tendency in the PBL, while a net spinup tendency exists over the central region.
The spindown tendency over the full domain in Nuri 3 must be a transient effect, as the overall circulation over the full domain increased between Nuri 3 and Nuri 4 in spite of the spindown tendency at the time of observation.

In the TCS030 case (Fig. 22) the circulation is almost nonexistent and the vertical mass flux is very weak. Curiously, the mass flux profile has a double maximum, with peak fluxes near $2 \mathrm{~km}$ and $8 \mathrm{~km}$, suggesting two different convective populations. Even though the entrained mass flux in a thin layer near the surface is quite large in spite of the weak overall profile, the vorticity convergence tendency in the PBL (and at all levels) is negative due to the export of vorticity. This export is related to the lack of a closed vorticity flux circulation in the PBL, as shown in Fig. 15. The circulation tendency due to tilting is positive in the upper troposphere.

\section{Discussion}

The formation of typhoon Nuri over a three day period was documented by aircraft missions on four successive days. During this period the cyclone evolved from a tropical wave to a tropical depression, a tropical storm, and finally to a 

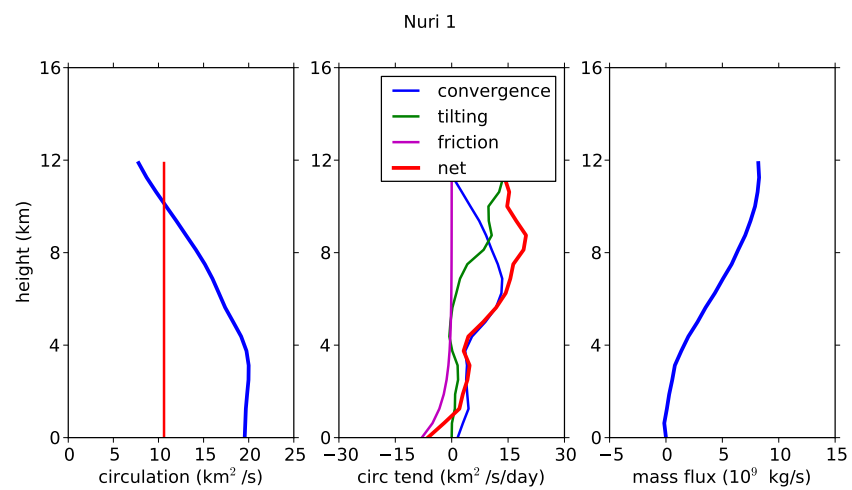

Fig. 16. Nuri 1 vertical profiles integrated over the analyzed region shown in Fig. 5. Left panel: Planetary (red) and absolute (blue) circulations. Center panel: Contributions to the total circulation tendency (red) due to vorticity convergence (blue), vortex tilting (green), and surface friction (magenta). Right panel: Vertical mass flux profile.
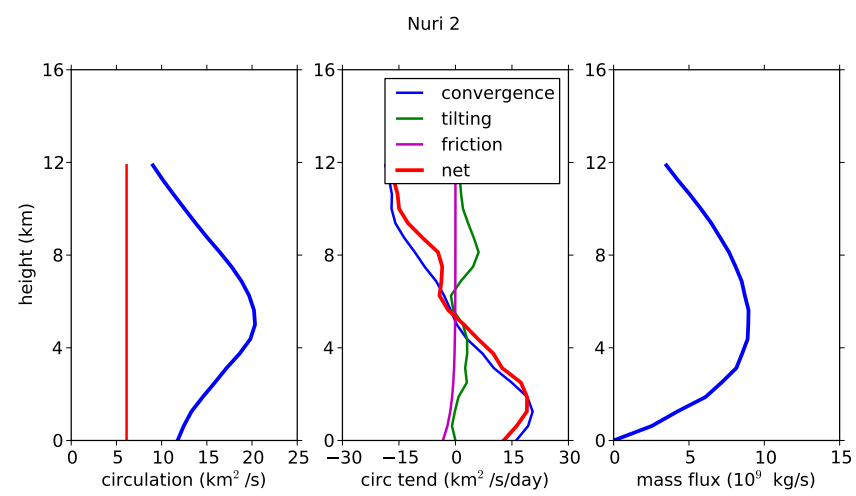

Fig. 17. As in Fig. 16 except Nuri 2 (Fig. 6).

full-fledged typhoon. This rapid intensification occurred in an environment of significant easterly and northeasterly shear (Fig. 11). Intensification began near the island of Guam. The storm followed a track toward the west during this period and Nuri was just to the east of the Philippines during the last mission (see Fig. 1).

As it evolved, Nuri's convection exhibited a variety of vertical mass flux profiles. In the tropical wave stage (Nuri 1) the average mass flux peaked at a high elevation, resulting in a deep inflow which peaks at middle levels. As a result, the circulation was decaying in the PBL, but increasing in the free troposphere. This is reflected in the greatly increased mid-level circulation seen in Nuri 2. While a tropical depression (Nuri 2), the vertical mass flux increased rapidly with height below $4 \mathrm{~km}$, resulting in an intense inflow in the PBL. This inflow was responsible for the strong positive circulation tendency associated with vorticity convergence at these levels. In the tropical storm stage (Nuri 3), the PBL inflow was less strong overall, resulting in a net spindown tendency in the PBL (see Fig. 18). However, as Fig. 19 shows, the

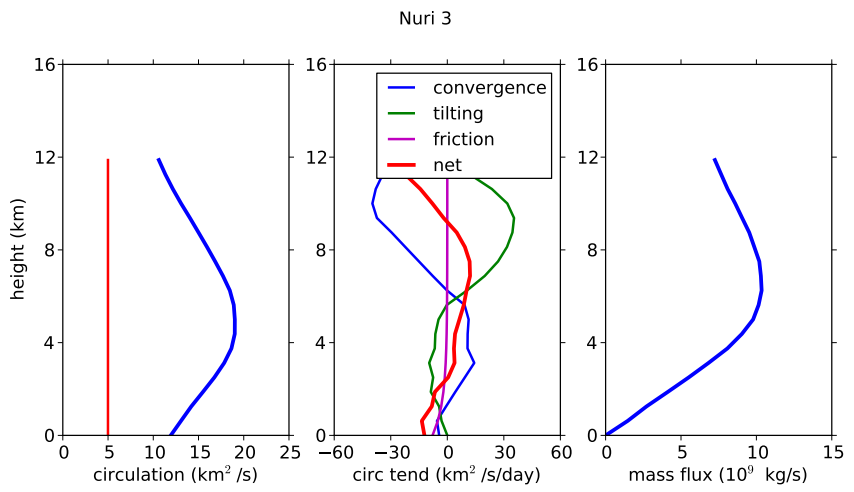

Fig. 18. As in Fig. 16 except Nuri 3 (Fig. 7). Note the change in scales.

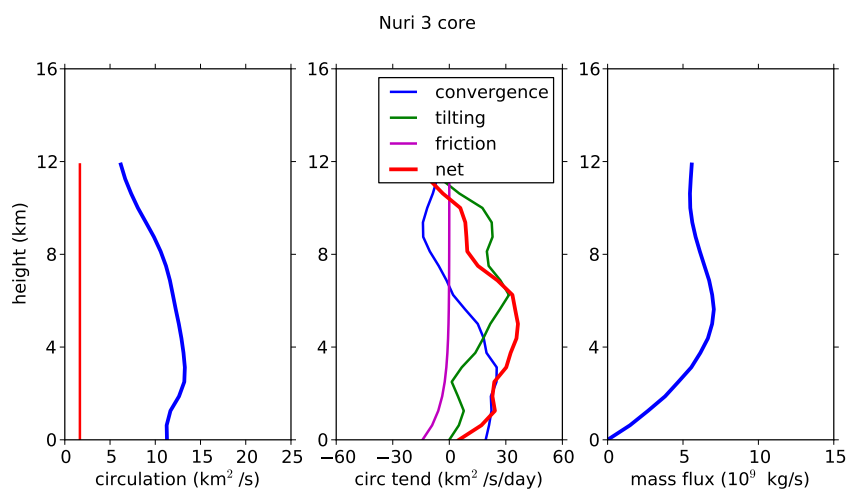

Fig. 19. As in Fig. 18 except for integration over the $2^{\circ}$-square box illustrated in Fig. 7. Note the change in scales.

spinup tendency around a small box centered on the circulation center remained intense. Figures 20 and 21 indicate that Nuri continued this trend as it developed into a typhoon.

In Nuri 1 and Nuri 2, the circulation centers in the PBL and at $5 \mathrm{~km}$ are displaced from each other by $2^{\circ}-3^{\circ}$. Comparison of Figs. 5 and 6 with the shears shown in Fig. 11 shows that the displacement of the $5 \mathrm{~km}$ circulation center relative to the PBL center is approximately $90^{\circ}$ to the left of the shear vector between the PBL and $5 \mathrm{~km}$. Thus, in the case of Nuri 1, the shear is from the north-northeast in the lowest $5 \mathrm{~km}$ and the $5 \mathrm{~km}$ circulation is southeast of the PBL circulation. For Nuri 2 the shear is east-northeasterly and the corresponding circulation center displacement is to the south-southeast.

Figure 23 gives a possible explanation for why the systemrelative circulation centers are displaced from each other. At each level the total wind is assumed to be the vector sum of the system-relative ambient wind and the induced circulation associated with the wave-scale region of positive relative vorticity, which in the idealization of Sect. 2.3 is approximated as an upright cylinder of vorticity which varies in magnitude only with height. The circulation center at each level occurs where the vector sum of these winds is zero. The 


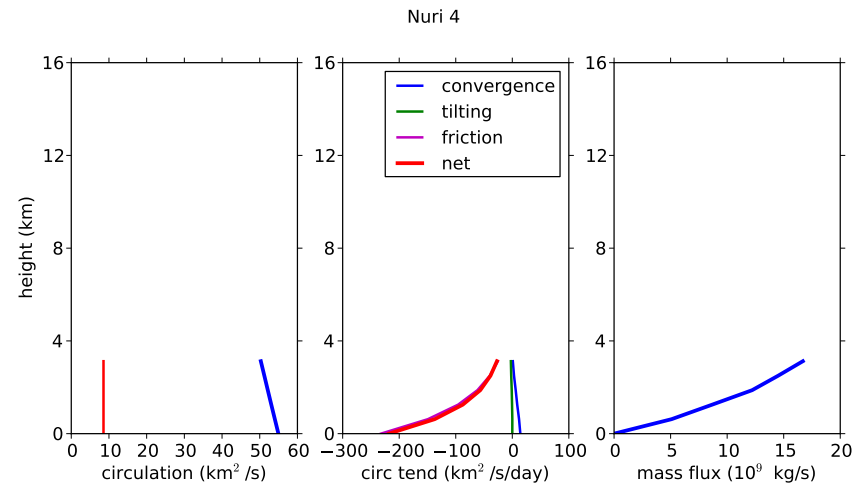

Fig. 20. As in Fig. 16 except Nuri 3 (Fig. 8). Note the change in scales.

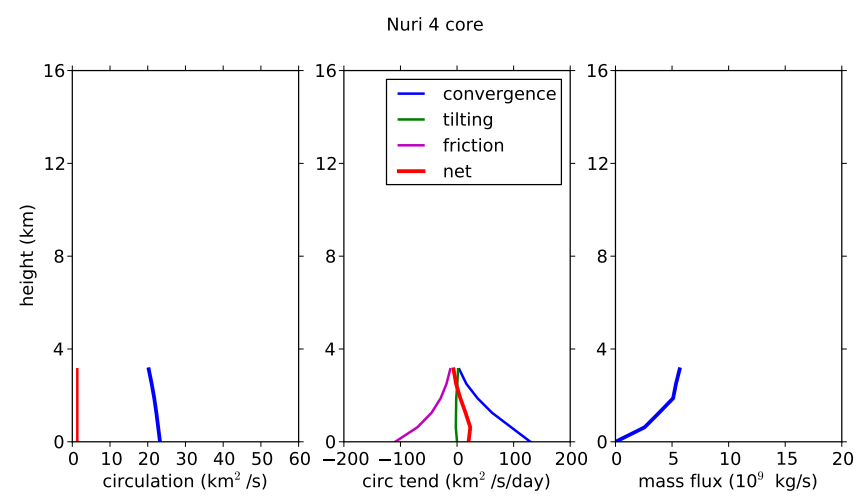

Fig. 21. As in Fig. 20 except for integration over the $1.5^{\circ}$-square box illustrated in Fig. 8. Note the change in scales.

displacement between the circulation centers is due to the difference in the ambient wind between the two levels, with the displacement being normal to this difference.

Around these circulation centers are closed regions of cyclonic flow as illustrated in Sect. 2.3. Air (and vorticity) at each level inside the bounding closed streamline remains within the system and is protected from entrainment of environmental air as postulated by Dunkerton et al. (2009) and Montgomery et al. (2010). The area where these regions overlap is protected from environmental incursions through the full column depth between the PBL and $5 \mathrm{~km}$. It is thus likely to be the area in which the core of the developing tropical cyclone spins up. In Nuri 1 this region contained the strongest deep convection. In Nuri 2 the most significant vorticity at $5 \mathrm{~km}$ was also found in this region, suggesting a history of strong convection there.

As discussed in Sect. 2.3, the above explanation for the displacement of the circulation center with height differs from explanations based on adiabatic vortex dynamics, which consider the interaction between potential vorticity patterns at different elevations. Our explanation depends on diabatic processes to maintain the form of the wave-scale vorticity pattern in the face of shear.

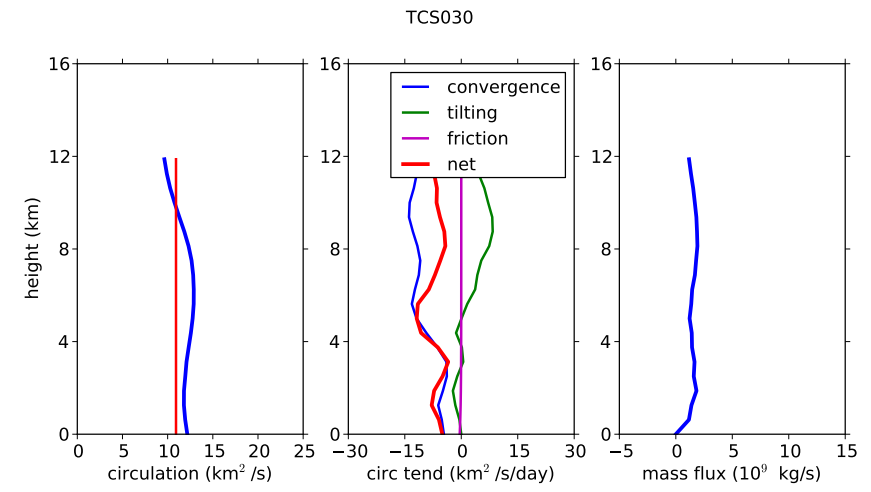

Fig. 22. As in Fig. 16 except TCS030 (Fig. 9).

The high resolution simulation of the development of Atlantic hurricane Felix (2007) by Wang et al. (2010a) and Wang et al. (2010b) shows many features similar to those seen in the formation of Nuri. In particular, the low-level spinup due to vorticity convergence driven by convection in a protected core and the spinup at upper levels by the tilting term are reproduced.

Nuri transformed from a pattern of scattered mesoscale vortices to a highly organized system with a strong central concentration of vorticity between mission 2 and mission 3 . However, this transformation was not sudden; examination of Figs. 12-14 shows that the number of regions of vortex stretching decreased as Nuri evolved and the strength of the stretching increased. This suggests the development of fewer but stronger rotating convective systems as Nuri intensified, culminating in a single core system which developed into the eyewall.

An interesting aspect of Nuri's evolution is that vorticity balance in the PBL was far from satisfied. In Nuri 1 and Nuri 3 (full observed region) the frictional spindown tendencies slightly exceeded the spinup tendencies due to vorticity convergence. In Nuri 2 spinup due to vorticity convergence far exceeded frictional spindown. Only in the restricted region encompassing the core of Nuri 3 was approximate vorticity balance observed (see Fig. 19). Uncertainties in the vertical distribution of surface stress are insufficient to explain this discrepancy, particularly for Nuri 2 . We note that significant imbalance was found in the boundary layer of a simulated, intensifying tropical storm (Bui et al., 2009) in agreement with our observations. The effects of tilting are generally insufficient to change these qualitative results, at least at low levels.

Superficially, the pre-Nuri tropical wave observed during Nuri 1 was similar to the wave seen in TCS030. Both cases appeared as tropical waves in large-scale analyses and both had similar values of shear. However, TCS030 did not exhibit a closed circulation at $5 \mathrm{~km}$ in our data and the existence of one in the PBL was doubtful, even in system-relative coordinates. Thus, Nuri was able to retain its moist core and 


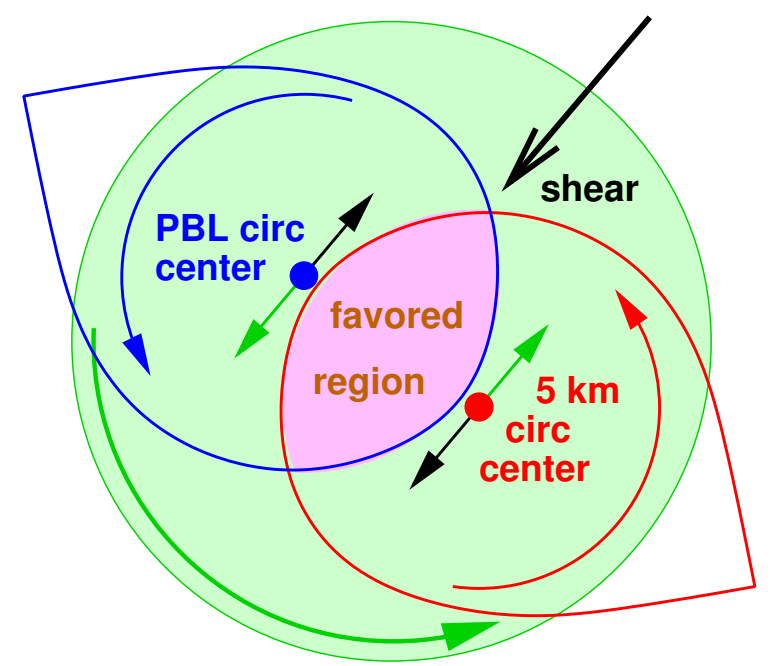

Fig. 23. Interaction of shear (thick black vector) with a broad region of positive relative vorticity (large green circle). The circulation centers in the PBL and at $5 \mathrm{~km}$ are located where the systemrelative ambient winds at the respective levels (black arrows) just balance the induced circulation from the vorticity pattern (green arrows). The blue and red "teardrops" represent the limits of closed circulation streamlines in the PBL and at $5 \mathrm{~km}$. The magentacolored region of overlap between these areas of closed circulation represents the region where the entire column between the PBL and $5 \mathrm{~km}$ is protected from incursions of environmental air.

vorticity over an extended period while TCS030 could not. The lack of a strong vertical mass flux profile in TCS030 most likely results from the lack of a protected core.

\section{Conclusions}

Our analyses of typhoon Nuri and tropical wave TCS030 support the following conclusions:

1. Nuri spun up rapidly from a tropical wave to a typhoon in spite of significant environmental shear. TCS030 was subject to similar vertical shear but did not spin up. As Nuri intensified, regions of convective vortex stretching became fewer and more intense, culminating in the formation of a strong central vortex.

2. The rapid intensification of Nuri appears to be related to the development of vertical mass flux profiles showing a strong increase with height below $4 \mathrm{~km}$ elevation. Mass continuity implies intense inflow at low levels in this case and correspondingly strong vorticity convergence. For Nuri 2, this vorticity convergence dominated all other circulation tendency terms in the planetary boundary layer, resulting in the development of Nuri into a tropical storm by the following day. The vertical mass flux in the non-developing TCS030 case was much weaker.
3. The displacement of the center of the Nuri circulation with height is explained as the result of the interaction with shear of a large, wave-associated column of positive relative vorticity. This displacement, though of order $2^{\circ}-3^{\circ}$ between the surface and $5 \mathrm{~km}$ in the wave and tropical depression stages, was still small enough for a column protected from environmental incursions to exist through this elevation range. Such a protected column did not exist for TCS030.

4. The planetary boundary layer was far from vorticity balance during Nuri's spinup.

This work complements the larger-scale view of Nuri presented by Montgomery et al. (2010).

Acknowledgements. We thank Mike Herman and Jorge Cisneros for the FNL and preliminary radar results; Chris Velden and Dave Stettner of SSEC for the MTSAT data; crews of the NRL P-3 and the Air Force Reserve WC-130Js for their excellent work; NCAR EOL crew for their dedication, especially in rebuilding ELDORA in the two weeks before the project and in dealing with severe dropsonde problems; and Pat Harr for his exemplary leadership. Quality control of dropsondes by EOL contributed to the fidelity of our analysis. We also thank Kerry Emanuel and Sharon Sessions for useful discussions on this manuscript. Roger Smith, Michael Montgomery, Kevin Tory, Zhuo Wang, and Michael Riemer made many penetrating and useful comments during the review process. This work was supported by grants N000140810241 from the Office of Naval Research and ATM0638801 from the US National Science Foundation. C. López was partially supported by Inter-American Institute for Global Change Research grant CRN-2048.

Edited by: T. J. Dunkerton

\section{References}

Bister, M. and Emanuel, K. A.: The genesis of hurricane Guillermo: TEXMEX analyses and a modeling study, Mon. Wea. Rev., 125, 2662-2682, 1997.

Black, P. G., D’Asaro, E. A., Drennan, W. M., French, J. R., Niiler, P. P., Sanford, T. B., Terrill, E. J., Walsh, E. J. and Zhang, J. A.: Air-sea exchange in hurricanes: Synthesis of observations from the coupled boundary layer air-sea transfer experiment, Bull. Am. Meteor. Soc., 88, 357-374, 2007.

Bui, H. H., Smith, R. K., Montgomery, M. T., and Peng, J.: Balanced and unbalanced aspects of tropical cyclone intensification, Quart. J. Roy. Meteor. Soc., 1715-1731, 2009.

Davis, C. A.: Piecewise potential vorticity inversion, J. Atmos. Sci., 49, 1397-1411, 1992.

Dunkerton, T. J., Montgomery, M. T., and Wang, Z.: Tropical cyclogenesis in a tropical wave critical layer: easterly waves, Atmos. Chem. Phys., 9, 5587-5646, doi:10.5194/acp-9-5587-2009, 2009.

Elsberry, R. L. and Harr, P. A.: Tropical cyclone structure (TCS08) field experiment science basis, observational platforms, and strategy, Asia-Pacific J. Atmos. Sci., 44, 209-231, 2008. 
Gray, W. M.: Global view of the origin of tropical disturbances and storms, Mon. Wea. Rev., 96, 669-700, 1968.

Haynes, P. H. and McIntyre, M. E.: On the evolution of vorticity and potential vorticity in the presence of diabatic heating and frictional or other forces, J. Atmos. Sci., 44, 828-841, 1987.

Hendricks, E. A., Montgomery, M. T. and Davis, C. A.: The role of vortical hot towers in the formation of tropical cyclone Diana (1984), J. Atmos. Sci., 61, 1209-1232, 2004.

Jones, S. C.: The evolution of vortices in vertical shear: I: Initially barotropic vortices, Quart. J. Roy. Meteor. Soc., 121, 821-851, 1995.

Jones, S. C.: The evolution of vortices in vertical shear: II: Largescale asymmetries, Quart. J. Roy. Meteor. Soc., 126, 3137-3159, 2000a.

Jones, S. C.: The evolution of vortices in vertical shear: III: Baroclinic vortices, Quart. J. Roy. Meteor. Soc., 126, 3161-3185, 2000 b.

López Carrillo, C., and Raymond, D. J.: Retrieval of threedimensional wind fields from Doppler radar data using an efficient two-step approach, Atmos. Meas. Tech. Discuss., 3, 44594495, doi:10.5194/amtd-3-4459-2010, 2010.

Mapes, B., and Houze, R. A. Jr.: Diabatic divergence profiles in western Pacific mesoscale convective systems, J. Atmos. Sci., 52, 1807-1828, 1995.

McBride, J. L., and Zehr, R.: Observational analysis of tropical cyclone formation. Part II: Comparison of nondeveloping versus developing systems, J. Atmos. Sci., 38, 1132-1151, 1981.

Montgomery, M. T., Nicholls, M. E., Cram, T. A. and Saunders, A. B.: A vortical hot tower route to tropical cyclogenesis, J. Atmos. Sci., 63, 355-386, 2006.

Montgomery, M. T., Lussier III, L. L., Moore, R. W., and Wang, Z.: The genesis of Typhoon Nuri as observed during the Tropical Cyclone Structure 2008 (TCS-08) field experiment - Part 1: The role of the easterly wave critical layer, Atmos. Chem. Phys., 10, 9879-9900, doi:10.5194/acp-10-9879-2010, 2010.
Nguyen, S. V., Smith, R. K., and Montgomery, M. T.: Tropicalcyclone intensification and predicability in three dimensions, Quart. J. Roy. Meteor. Soc., 134, 563-582, 2008.

Raymond, D. J.: Nonlinear balance and potential-vorticity thinking at large Rossby number, Quart. J. Roy. Meteor. Soc., 118, 9871015, 1992.

Raymond, D. J. and Sessions, S. L.: Evolution of convection during tropical cyclogenesis, Geophys. Res. Lett., 34, L06811, doi:10.1029/2006GL028607, 2007.

Raymond, D. J., López-Carrillo, C. and López Cavazos, L.: Casestudies of developing east Pacific easterly waves, Quart. J. Roy. Meteor. Soc., 124, 2005-2034, 1998.

Reasor, P. D. and Montgomery, M. T.: Three-dimensional alignment and corotation of weak, TC-like vortices via linear vortex Rossby waves, J. Atmos. Sci., 58, 2306-2330, 2001.

Reasor, P. D., Montgomery, M. T., and Grasso, L. D.: A new look at the problem of tropical cyclones in vertical shear flow: Vortex resiliency, J. Atmos. Sci., 61, 3-22, 2004.

Reynolds, R. W. and Marsico, D. C.: An improved real-time global sea surface temperature analysis, J. Climate, 6, 114-119, 1993.

Ritchie, E. A. and Holland, G. J.: Scale interactions during the formation of Typhoon Irving, Mon. Wea. Rev., 125, 1377-1396, 1997.

Simpson, J., Ritchie, E., Holland, G. J., Halverson, J., and Stewart, S.: Mesoscale interactions in tropical cyclone genesis, Mon. Wea. Rev., 125, 2643-2661, 1997.

Smith, R. K. and Montgomery, M. T.: Balanced boundary layers used in hurricane models, Quart. J. Roy. Meteor. Soc., 134, 1385-1395, 2008.

Wang, Z., Montgomery, M. T., and Dunkerton, T. J.: Genesis of pre-hurricane Felix (2007). Part I: The role of the easterly wave critical layer, J. Atmos. Sci., 67, 1711-1729, 2010a.

Wang, Z., Montgomery, M. T., and Dunkerton, T. J.: Genesis of prehurricane Felix (2007). Part II: Warm core formation, precipitation evolution, and predictability, J. Atmos. Sci., 67, 1730-1744, 2010b. 